\title{
Exponentials and Logarithms Properties in an Extended Complex Number Field
}

\author{
Daniel Tischhauser
}

September 10th, 2021

\begin{abstract}
It is well established the complex exponential and logarithm are multivalued functions, both failing to maintain most identities originally valid over the positive integers domain. Moreover the general case of complex logarithm, with a complex base, is hardly mentionned in mathematic litterature. We study the exponentiation and logarithm as binary operations where all operands are complex. In a redefined complex number system using an extension of the $\mathbb{C}$ field, hereafter named $\mathbb{E}$, we proove both operations always produce single value results and maintain the validity of identities such as $\log _{u}(w v)=\log _{u}(w)+\log _{u}(v)$ where $u, v, w \in \mathbb{E}$. There is a cost as some algebraic properties of the addition and subtraction will be diminished, though remaining valid to a certain extent. In order to handle formulas in a $\mathbb{C}$ and $\mathbb{E}$ dual number system, we introduce the notion of set precision and set truncation. We show complex numbers as defined in $\mathbb{C}$ are insufficiently precise to grasp all subtleties of some complex operations, as a result multivaluation, identity failures and, in specific cases, wrong results are obtained when computing exclusively in $\mathbb{C}$. A geometric representation of the new complex number system is proposed, in which the complex plane appears as an orthogonal projection, and where the complex logarithm an exponentiation can be simply represented. Finally we attempt an algebraic formalization of $\mathbb{E}$.
\end{abstract}

Keywords: Complex number field; Complex exponentiation; Complex logarithm; Exponential and logarithm identities

\section{Introduction}

In 1749 L. Euler [1] solved a decades old controversy between G.W. Leibniz and J. Bernoulli over the appropriate definition for logarithms of negative and imaginary values, by producing the formula $\ln (z)=\ln (a+b i)=\ln |z|+\arg (z) i=\ln |z|+\theta i+2 k \pi i$, where $|z|=\sqrt{a^{2}+b^{2}}, \theta$ the principal value of $\arg (z), k \in \mathbb{Z}$.

The formula for complex exponentiation $z^{w}=(a+b i)^{m+n i}=x+y i$, where both $z, w \in \mathbb{C}$, was also given the same year by L.Euler in another study [2].

$$
\begin{aligned}
z^{w} & =e^{w \ln z}=\left(e^{l n|z|+\theta i+2 k \pi i}\right)^{m+n i}=e^{m \ln |z|-n \theta-n 2 k \pi} e^{(n \ln |z|+m \theta+m 2 k \pi) i} \\
x & =|z|^{m} e^{-n \theta-n 2 k \pi} \cos (n \ln |z|+m \theta+m 2 k \pi)
\end{aligned}
$$




$$
y=|z|^{m} e^{-n \theta-n 2 k \pi} \sin (n \ln |z|+m \theta+m 2 k \pi)
$$

The formula 1.1 produces an infinite number of results, depending on the value of the $k$ integer. Due to the periodicity of the sine and cosine functions, the results reduce to a finite number of results if the exponent $w \in \mathbb{Q}$.

The first complex logarithm formula $\log _{z} w=x+y i$, where both $z, w \in \mathbb{C}$, was given by M. Ohm in 1829 [3].

$$
\begin{aligned}
\log _{z} w & =\frac{\ln w}{\ln z}=\frac{\ln |w|+\theta_{w} i+2 k_{w} \pi i}{\ln |z|+\theta_{z} i+2 k_{z} \pi i} \\
x & =\frac{\ln |w| \ln |z|+\left(\theta_{w}+2 k_{w} \pi\right)\left(\theta_{z}+2 k_{z} \pi\right)}{(\ln |z|)^{2}+\left(\theta_{z}+2 k_{z} \pi\right)^{2}} \\
y & =\frac{\ln |z|\left(\theta_{w}+2 k_{w} \pi\right)-\ln |w|\left(\theta_{z}+2 k_{z} \pi\right)}{(\ln |z|)^{2}+\left(\theta_{z}+2 k_{z} \pi\right)^{2}}
\end{aligned}
$$

The formula 1.4 produces an infinite number of results, depending on both $k_{z}$ and $k_{w}$ integers. The formula is hardly mentionned in mathematic literature. In 1921, F. Cajori in his History of exponentials and logarithms [4] expressed it this way :

The general logarithm system failed of recognition as useful mathematical inventions.

Both general complex exponentiation and logarithm formulas are nevertheless used by complex number calculators, though usually only the principal value at $k=k_{z}=k_{w}=0$ is returned. By combining formulas 1.1 and 1.4, it is easily verified the complex logarithm is the reciprocal of the exponentiation within a particular branch.

$$
\begin{aligned}
\log _{z}\left(z^{w}\right)=\frac{\ln \left(z^{w}\right)}{\ln (z)} & =\frac{\left(m \ln |z|-n \theta_{z}-n 2 k \pi\right)+\left(n \ln |z|+m \theta_{z}+m 2 k \pi\right) i}{\ln |z|+\theta_{z} i+2 k_{z} \pi i} \\
& =\frac{(m+n i) \ln |z|+(m+n i) \theta_{z} i+(m+n i) 2 k \pi i}{\ln |z|+\theta_{z} i+2 k_{z} \pi i} \\
& =m+n i=w \quad\left(\text { when } k=k_{z}\right)
\end{aligned}
$$

In the same volume M. Ohm [3] studies the validity of the exponential and logarithm identities in $\mathbb{C}$. He concludes the set of values on both sides of the identity equation can differ. As an example the left side of $\left(z^{w}\right)^{v}=z^{w v}$ will produce many more results than the right side, since exponentiation is performed twice. Furthermore many identities such as $\ln (w v)=\ln (w)+\ln (v)$ are not always valid when considering the principal value or any other branch.

The formulas of Euler and Ohm show that all results of exponentiation and logarithm can be expressed in the form $x+y i$. Thus both operations are algebraically closed in $\mathbb{C}$ and can be defined either as multivalued functions or, when considering a particular branch, as ordinary functions $f: \mathbb{C} \times \mathbb{C} \rightarrow \mathbb{C}$. However the closure has come at a cost, firstly most identities equations valid in $\mathbb{R}_{+}$fail in $\mathbb{C}$, secondly the multivaluation forces an arbitrary selection of a branch prior to any result evaluation. 
In tables 1 and 2 we summarize the validity in $\mathbb{R}_{+}$and $\mathbb{C}$ of the exponentiation and logarithm main properties and identities.

Table 1: Exponentiation and logarithm properties.

\begin{tabular}{lcc}
\hline Property & Validity in $\mathbb{R}_{+}$ & Validity in $\mathbb{C}$ \\
\hline Exponentiation $z_{1}^{z_{2}}$ closure & yes & yes \\
Logarithm $\log _{z_{1}}\left(z_{2}\right)$ closure & no & yes \\
Exponentiation monovaluation & yes & no \\
Logarithm monovaluation & yes & no \\
Exponentiation inverse of logarithm & & subset $^{1}$ \\
$z_{1} \log _{z 1}\left(z_{2}\right)=z_{2}$ & yes & \\
$\operatorname{Logarithm}$ inverse of exponentiation & & subset $^{1}$ \\
$\log _{z_{1}}\left(z_{1} z_{2}\right)=z_{2}$ & yes
\end{tabular}

${ }^{1}$ The left side of the equation produces many more results, of which only a subset is equal to the right side. The equation always holds at principal value

Table 2: Exponentiation and logarithm identities.

\begin{tabular}{|c|c|c|}
\hline Identity & Validity in $\mathbb{R}_{+}$ & Validity in $\mathbb{C}$ \\
\hline $\begin{array}{l}\text { Exponent distributivity over multiplication } \\
\left(z_{1} z_{2}\right)^{z_{3}}=z_{1} z_{3} z_{2}^{z_{3}}\end{array}$ & yes & no ${ }^{2}$ \\
\hline $\begin{array}{l}\text { Exponent distributivity over division } \\
\left(\frac{z_{1}}{z_{2}}\right)^{z_{3}}=\frac{z_{1} z_{3}}{z_{2} z_{3}}\end{array}$ & yes & no ${ }^{2}$ \\
\hline Exponential product $z_{1}^{z_{2}} z_{1}^{z_{3}}=z_{1}^{z_{2}+z_{3}}$ & yes & subset $^{1}$ \\
\hline Exponential quotient $\frac{z_{1}^{z_{2}}}{z_{1} z_{3}}=z_{1}^{z_{2}-z_{3}}$ & yes & subset $^{1}$ \\
\hline Exponential power $\left(z_{1}^{z_{2}}\right)^{z_{3}}=z_{1}^{z_{2} z_{3}}$ & yes & no ${ }^{2}$ \\
\hline $\begin{array}{l}\text { Logarithm product } \\
\log _{z_{1}}\left(z_{2} z_{3}\right)=\log _{z_{1}}\left(z_{2}\right)+\log _{z_{1}}\left(z_{3}\right)\end{array}$ & yes & no ${ }^{2}$ \\
\hline $\begin{array}{l}\text { Logarithm quotient } \\
\log _{z_{1}}\left(\frac{z_{2}}{z_{3}}\right)=\log _{z_{1}}\left(z_{2}\right)-\log _{z_{1}}\left(z_{3}\right)\end{array}$ & yes & no ${ }^{2}$ \\
\hline Logarithm power $\log _{z_{1}}\left(z_{2} z_{3}\right)=z_{3} \log _{z_{1}}\left(z_{2}\right)$ & yes & no ${ }^{2}$ \\
\hline $\begin{array}{l}\text { Logarithm base substitution } \\
\log _{z_{1}}\left(z_{2}\right)=\frac{\log _{z_{3}}\left(z_{1}\right)}{\log _{z_{3}}\left(z_{2}\right)}\end{array}$ & yes & yes, if $z_{3} \in \mathbb{R}_{+}$ \\
\hline
\end{tabular}

${ }^{1}$ The left side of the equation produces many more results, of which only a subset is equal to the right side. The equation always holds at principal value

${ }^{2}$ Both sides of identities equations produce a different set of results, which are not necessarily equal at the principal value. In further sections we give the exact conditions for the identity validity at the principal value or at any given branch 


\section{Trivial examples of identity failures in $\mathbb{C}$}

For clarity only the principal value at $k=0$ is considered, the same outcome occurs on other branches.

$$
\begin{aligned}
& (-1 \cdot-1)^{\frac{1}{2}}=(1)^{\frac{1}{2}}=1 \neq(-1)^{\frac{1}{2}} \cdot(-1)^{\frac{1}{2}}=i \cdot i=-1 \\
& (i-1)^{2 i}=\left(\sqrt{2} e^{\frac{3 \pi}{4}} i\right)^{2 i}=2^{i} e^{-\frac{3 \pi}{2}} \neq((i-1)(i-1))^{i}=(-2 i)^{i}=\left(2 e^{-\frac{\pi}{2} i}\right)^{i}=2^{i} e^{\frac{\pi}{2}} \\
& \ln \left((-i)^{2}\right)=\ln (-1)=\pi i \neq 2 \ln (-i)=2\left(-\frac{\pi}{2} i\right)=-\pi i \\
& \log _{-2}\left((-2)^{5}\right)=\log _{-2}(-32)=\frac{\ln (-32)}{\ln (-2)}=\frac{\ln (32)+\pi i}{\ln (2)+\pi i}=\frac{(\ln (32)+\pi i)(\ln (2)-\pi i)}{(\ln (2)+\pi i)(\ln (2)-\pi i)} \\
& =\frac{\ln (32) \ln (2)+(\ln (32)-\ln (2)) \pi i+\pi^{2}}{(\ln (2))^{2}+\pi^{2}}=1.18568 \ldots+0.84157 \ldots i \\
& \neq 5 \log _{-2}(-2)=5 \\
& \ln (-1 \cdot i)=\ln (-i)=-\frac{\pi}{2} i \neq \ln (-1)+\ln (i)=\pi i+\frac{\pi}{2} i=\frac{3 \pi}{2} i
\end{aligned}
$$

The aim of this article is to propose a redefinition of the complex number set in which the issues described above resolve. The idea is to introduce a new form of complex number that extends the possibilities of the algebraic form, since the latter will prove insufficient to grasp all the subtleties of the exponentiation and logarithm when all operands are complex. The properties of the basic operations $(+,-, \times, \div)$ will be impacted by the redefinition.

The sections 2 and 3 are dedicated to the definition of a new complex number set, hereafter named $\mathbb{E}$, the equivalences between $\mathbb{C}$ and $\mathbb{E}$, and to the definition of complex operations $(+,-, \times, \div, \exp , \log )$ in $\mathbb{E}$. The exponentiation is no longer defined by the logarithm, instead the complex logarithm formula can be deduced from the exponentiation. Moreover all operations produce a single value result.

The section 4 includes all proofs and some examples over the validity of the exponential and logarithm identities in $\mathbb{E}$. All the trivial identity failure cases given above resolve.

In the section 5 we show how to obtain explicit formulas linking the real and imaginary parts of some transcendental equations solutions.

The section 6 proposes a geometric representation of $\mathbb{E}$, of which the complex plane appears as an orthogonal projection. The complex exponentiation $z=z_{1} z_{2}$ and logarithm $z=\log _{z_{1}}\left(z_{2}\right)$, where $z, z_{1}, z_{2} \in \mathbb{E}$, can be simply represented as a mapping of the two operands elements to the result element.

The section 7 lists all algebraic properties of $\mathbb{E}$ and compares them with the properties of the $\mathbb{R}$ and $\mathbb{C}$ fields.

In section 8 we argue why the exponentiation and logarithm multivalued results and identity failures in $\mathbb{C}$ are not induced by the operations, but are induced by an intrinsic limitation of the complex numbers algebraic form $z=x+y i$. 


\section{Complex numbers in complete form}

Definition 1. Complex number in complete form

By taking a broad definition of the complex number as a number composed of a real part and an imaginary part, the complete complex number set is defined as all numbers in the form $e^{a} e^{b i}, \cup\{0\}$, where $a, b \in \mathbb{R}$ and $i^{2}=-1$

The number set is hereafter named $\mathbb{E}$. The real part is defined as $e^{a}$ and the imaginary part $e^{b i}$, where $a$ is the real argument and $b$ the imaginary argument. The element 0 is included for compatibility with $\mathbb{C}$ and $\mathbb{R}$.

\section{Remark. Equivalence with the exponential form}

The exponential form of complex numbers $z=x+y i=|z| e^{\arg (z) i}=|z| e^{\theta i+2 k \pi i}$ has a similar but not identical definition. It remains explicitly linked to the algebraic form and must have a principal value $\theta$ of the $\operatorname{argument} \arg (z)$ within the interval $]-\pi ; \pi]$. The purpose of the integer $\mathrm{k}$ is precisely to link all values of the exponential form to their unique corresponding algebraic form. Geometrically, the $2 \pi$ periodicity of the imaginary argument is purposely maintaining the correlation with the complex plane.

In the complete form, the explicit link to the algebraic form and the constraint on the argument principal value are abolished. For example in $\mathbb{E}$ the numbers $e^{0} e^{2 \pi i}$ and $e^{0} e^{4 \pi i}$ are not equal, each having distinct properties as it will be demonstrated in further sections. Within $\mathbb{C}$ the symbolic and geometric representation of both numbers are equally represented by 1 and by the coordinates $(x, y)=(1,0)$ on the complex plane.

Replacing $|z|$ by $e^{a}$ allows the establishment of more elegant and symmetrical formulas. We use the new denomination complete form to avoid any ambiguity.

\section{Definition 2. Set partitions of $\mathbb{E}$}

Let the set $\mathbb{E}$ of complex numbers in complete form $e^{a} e^{b i}$ be partitionned into $\mathbb{C}$ and $\mathbb{E} \backslash \mathbb{C}$ by restricting $\mathbb{C}$ to a $2 \pi$ interval of the imaginary argument b, by convention the interval $b \in]-\pi ; \pi]$. Each number $x+y i \in \mathbb{C}$ converted into its unique corresponding complete form $e^{a} e^{b i}$ forms then a distinct equivalence class together with numbers in the form $e^{a} e^{(b+2 k \pi) i} \in \mathbb{E}$ with $k \in \mathbb{Z}^{*}$.

The definition is equivalent as restricting $\mathbb{C}$ to the principal value of the exponential form of complex numbers. Even with this restriction, the algebraic definition of $\mathbb{C}$ and the complex plane definition are not altered.

\section{Definition 3. Set precision and truncation}

Let $A$ be a set partitionned by an equivalence relation into two subsets $A_{1}$ and $A_{2}$, and let each element $a_{1} \in A_{1}$ form a distinct equivalence class with an arbitrary number of elements $a_{2} \in A_{2}$ such as each element $a$ is part of a unique given class. In such a set configuration, elements $a_{2}$ are defined as $A$ precise, elements $a_{1}$ are defined as $A_{1}$ precise. 
Each element $a_{2} \in A_{2}$ can be truncated to its unique corresponding $a_{1} \in A_{1}$ element, thus at a lower precision level. The truncation is noted $a_{1}=\left|a_{2}\right|_{A_{1}}$.

\section{Example 1. $\mathbb{Z}$ and $\mathbb{N}$ precision}

Let the integer set $\mathbb{Z}$ be partitionned into $\mathbb{N}$ and $\mathbb{Z}_{<0}$, an integer is $\mathbb{Z}$ precise if negative, and is $\mathbb{N}$ precise if positive or zero. The abs function is the truncation function from $\mathbb{Z}$ to $\mathbb{N}$ precision level.

\section{Example 2. $\mathbb{E}$ and $\mathbb{C}$ precision}

The Euler formula $e^{b i}=\cos b+\sin b i$ is de facto the truncation function from $\mathbb{E}$ to $\mathbb{C}$ precision. The truncation can be noted $|z|_{\mathbb{C}}=\left|e^{a} e^{b i}\right|_{\mathbb{C}}=e^{a} \cos b+e^{a} \sin b i=e^{a} e^{|b|_{c} i}$, with the imaginary argument truncated such as :

$|b|_{\mathbb{C}}= \begin{cases}b(\bmod 2 \pi) & \text { if } b(\bmod 2 \pi)<=\pi \\ b(\bmod 2 \pi)-2 \pi & \text { if } b(\bmod 2 \pi)>\pi\end{cases}$

Equalities such as $1=e^{4 \pi i}$ or $1=e^{2 k \pi i}$ no longer hold whenever $\mathbb{E}$ precision is required, the notation $\left|e^{2 k \pi i}\right|_{\mathbb{C}}=e^{0 i}=1$ can be used to clearly indicate the truncation.

\section{Remark.}

The $\mathbb{E}$ set of complex numbers can be viewed as a "natural" extension of $\mathbb{C}$. Within the set sequence $\mathbb{N} \subset \mathbb{Z} \subset \mathbb{R} \subset \mathbb{C} \subset \mathbb{E}$ each element in a given set is uniquely linked to a predecessor set element through an equivalence relation, therefore an element can always be truncated to the predecessor set precision level.

Lemma 1. Converting from complete form to algebraic form

$$
\begin{aligned}
& z=e^{a} e^{b i} \Longrightarrow e^{a} \cos b+e^{a} \sin b i=x+y i \\
& x=e^{a} \cos b \\
& y=e^{a} \sin b
\end{aligned}
$$

The Euler formula used for the conversion is not to be considered as an equality. From a $\mathbb{E}$ perspective an irreversible loss of information is induced when converting from complete to algebraic form if the imaginary argument is outside the interval ] $-\pi ; \pi]$.

\section{Lemma 2. Converting from algebraic form to complete form}

Using the definition of complex number modulus and argument. By definition $z=0$ is equivalent in $\mathbb{E}$ and $\mathbb{C}$.

$$
\begin{aligned}
z & =x+y i=|z| e^{\operatorname{Arg}(z) i}=e^{\ln |z|} e^{\theta_{z} i} \Longrightarrow e^{\frac{1}{2} \ln \left(x^{2}+y^{2}\right)} e^{\operatorname{Atan}\left(\frac{y}{x}\right) i}=e^{a} e^{b i} \\
a & =\frac{1}{2} \ln \left(x^{2}+y^{2}\right) \\
b & =\operatorname{Atan}\left(\frac{y}{x}\right)
\end{aligned}
$$


Remark. Usage of $\ln$, Arg and Atan functions

The natural logarithm function is applied to the domain $\mathbb{R}_{>0}$, hence is single valued. In the formula 2.4 only the principal value of the arg function is considered to remain consistent with definition 2. The limits of the traditional arctan function, with the result in the interval ] $\left.-\frac{\pi}{2} ; \frac{\pi}{2}\right]$, requires the use of the atan2 function with 2 arguments whose result is included in the interval ] $-\pi ; \pi]$ without singularities. In this study the notation $\operatorname{Atan}\left(\frac{y}{x}\right)$ always refers to the atan2 function where both arguments remain as the fraction numerator and denominator. This notation adjustment will ease the readibility and handling of formulas, as obtained formulas always produce a fraction inside the Atan argument. The fraction can be simplified providing the numerator and denominator signum are preserved.

\section{Binary operations in complete form}

Definition 4. Complex binary operations in $\mathbb{E}$

The operations in $\mathbb{E}$ are defined as the 4 basic operations,,$+- \times, \div$ together with exponentiation and logarithm. All operands and results are expressed in complete form.

\section{Lemma 3. Formulas for operations computation in $\mathbb{E}$}

Similarly as in $\mathbb{C}$, the operations computation use a combination of real functions and real operations $(+,-, \times, \div, \exp , \ln , \sin , \cos$, atan 2$)$. Let $z_{1}=e^{a_{1}} e^{b_{1} i}$ and $z_{2}=e^{a_{2}} e^{b_{2} i}$

$$
\begin{aligned}
& z_{1} \times z_{2}=e^{a_{1}+a_{2}} e^{\left(b_{1}+b_{2}\right) i} \\
& z_{1} \div z_{2}=e^{a_{1}-a_{2}} e^{\left(b_{1}-b_{2}\right) i}
\end{aligned}
$$

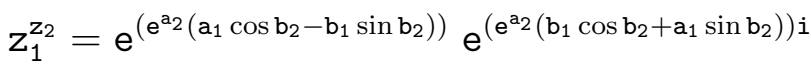

$$
\begin{aligned}
& \log _{z_{1}} z_{2}=e^{\frac{1}{2} \ln \frac{a_{2}{ }^{2}+b_{2}{ }^{2}}{a_{1}{ }^{2}+b_{1}{ }^{2}}} e^{\operatorname{Atan} \frac{a_{1} b_{2}-a_{2} b_{1}}{a_{1} a_{2}+b_{1} b_{2}} i} \\
& z_{1}+z_{2}=e^{\frac{1}{2} \ln \left(e^{2 a_{1}}+e^{2 a_{2}}+2 e^{a_{1}+a_{2}} \cos \left(b_{1}-b_{2}\right)\right)} e^{A \tan \frac{e^{a_{1}} \sin b_{1}+e^{a_{2}} \sin b_{2}}{e^{a_{1}} \cos b_{1}+e^{a_{2}} \cos b_{2}} i} \\
& z_{1}-z_{2}=e^{\frac{1}{2} \ln \left(e^{2 a_{1}}+e^{2 a_{2}}-2 e^{a_{1}+a_{2}} \cos \left(b_{1}-b_{2}\right)\right)} e^{A \tan \frac{e^{a_{1}} \sin b_{1}-e^{a_{2}} \sin b_{2}}{e^{a_{1}} \cos b_{1}-e^{a_{2}} \cos b_{2}} i}
\end{aligned}
$$

Formulas are easier to handle when split between real and imaginary parts, in this study we mostly use the split notation. Let $z=e^{a} e^{b i}$ :

$$
\begin{aligned}
& z=z_{1} \times z_{2} \\
& z=z_{1} \div z_{2} \\
& a=a_{1}+a_{2} \\
& a=a_{1}-b_{1} \\
& b=b_{1}+b_{2} \\
& b=b_{1}-b_{2} \\
& z=z_{1}^{z_{2}} \\
& z=\log _{z_{1}} z_{2} \\
& a=e^{a_{2}}\left(a_{1} \cos b_{2}-b_{1} \sin b_{2}\right) \\
& a=\frac{1}{2} \ln \left(\frac{a_{2}^{2}+b_{2}^{2}}{a_{1}^{2}+b_{1}^{2}}\right) \\
& b=e^{a_{2}}\left(b_{1} \cos b_{2}+a_{1} \sin b_{2}\right) \\
& b=\operatorname{Atan}\left(\frac{a_{1} b_{2}-a_{2} b_{1}}{a_{1} a_{2}+b_{1} b_{2}}\right)
\end{aligned}
$$




$$
\begin{array}{rlrl}
z & =z_{1}+z_{2} & z & =z_{1}-z_{2} \\
a & =\frac{1}{2} \ln \left(e^{2 a_{1}}+e^{2 a_{2}}+2 e^{a_{1}+a_{2}} \cos \left(b_{1}-b_{2}\right)\right) & a & =\frac{1}{2} \ln \left(e^{2 a_{1}}+e^{2 a_{2}}-2 e^{a_{1}+a_{2}} \cos \left(b_{1}-b_{2}\right)\right) \\
b & =\operatorname{Atan}\left(\frac{e^{a_{1}} \sin b_{1}+e^{a_{2}} \sin b_{2}}{e^{a_{1}} \cos b_{1}+e^{a_{2}} \cos b_{2}}\right) & b & =\operatorname{Atan}\left(\frac{e^{a_{1}} \sin b_{1}-e^{a_{2}} \sin b_{2}}{e^{a_{1}} \cos b_{1}-e^{a_{2}} \cos b_{2}}\right)
\end{array}
$$

Proof. Multiplication formula

Using the identity $e^{w_{1}} \cdot e^{w_{2}}=e^{w_{1}+w_{2}}$ where $w_{1}, w_{2} \in \mathbb{C}[7]$

$$
\begin{aligned}
& z=z_{1} \times z_{2}=e^{a_{1}} e^{b_{1} i} \cdot e^{a_{2}} e^{b_{2} i}=e^{a_{1}} e^{a_{2}} e^{b_{1} i} e^{b_{2} i}=e^{a_{1}+a_{2}} e^{\left(b_{1}+b_{2}\right) i} \\
& a=a_{1}+a_{2} \\
& b=b_{1}+b_{2}
\end{aligned}
$$

\section{Proof. Division formula}

Using the identity $e^{w_{1}} / e^{w_{2}}=e^{w_{1}-w_{2}}$ where $w_{1}, w_{2} \in \mathbb{C}[7]$

$$
\begin{aligned}
& z=z_{1} \div z_{2}=\frac{e^{a_{1}} e^{b_{1} i}}{e^{a_{2}} e^{b_{2} i}}=\frac{e^{a_{1}}}{e^{a_{2}}} \cdot \frac{e^{b_{1} i}}{e^{b_{2} i}}=e^{a_{1}-a_{2}} e^{\left(b_{1}-b_{2}\right) i} \\
& a=a_{1}-a_{2} \\
& b=b_{1}-b_{2}
\end{aligned}
$$

\section{Proof. Exponentiation formula}

The formula $u^{w}=e^{w \ln u}$ with $w, u \in \mathbb{C}$ defines the complex exponentiation in $\mathbb{C}$, the formula is necessary given the base cannot be exploited directly in algebraic form. The formula is equivalent as converting the base into an infinity of bases in the form $u=$ $e^{\ln |u|+\theta i+2 k \pi i}$. The exponent is then applied to the bases such as $u^{w}=\left(e^{\ln |u|+\theta i+2 k \pi i}\right)^{w}=$ $e^{w \ln |u|+w(\theta+2 k \pi) i}$. The result is then reconverted into algebraic form. When calculated separately for each integer $\mathrm{k}$, the exponentiation can be defined as $\left(e^{a} e^{b i}\right)^{w}=e^{a w} e^{b w i}$ with a single valued result, the base and result being in complete form and the exponent in algebraic form. Let $z_{1}=e^{a_{1}} e^{b_{1} i}$ and, using the conversion formula 2.1, let $z_{2}=e^{a_{2}} e^{b_{2} i} \Longrightarrow$ $e^{a_{2}} \cos b_{2}+e^{a_{2}} \sin b_{2} i$.

$$
\begin{aligned}
z & =z_{1}^{z_{2}}=\left(e^{a_{1}} e^{b_{1} i}\right)^{\left(e^{a_{2}} \cos b_{2}+e^{a_{2}} \sin b_{2} i\right)} \\
& =\left(e^{a_{1}+b_{1} i}\right)^{\left(e^{a_{2}} \cos b_{2}+e^{a_{2}} \sin b_{2} i\right)} \\
& =e^{\left(a_{1}+b_{1} i\right)\left(e^{a_{2}} \cos b_{2}+e^{a_{2}} \sin b_{2} i\right)} \\
& =e^{\left(a_{1} e^{a_{2}} \cos b_{2}+a_{1} e^{a_{2}} \sin b_{2} i+b_{1} e^{a_{2}} \cos b_{2} i-b_{1} e^{a_{2}} \sin b_{2}\right)} \\
& =e^{e^{a_{2}}\left(a_{1} \cos b_{2}-b_{1} \sin b_{2}\right)} e^{e_{2}\left(b_{1} \cos b_{2}+a_{1} \sin b_{2}\right) i} \\
a & =e^{a_{2}}\left(a_{1} \cos b_{2}-b_{1} \sin b_{2}\right) \\
b & =e^{a_{2}}\left(b_{1} \cos b_{2}+a_{1} \sin b_{2}\right)
\end{aligned}
$$

Proof. Logarithm formula 
The logarithm formula can be directly reversed from the exponentiation formula 3.3. Counter to the definition of the complex logarithm in $\mathbb{C}$, both operands are here in $\mathbb{E}$ thus can be exploited directly in the formula without requiring any conversion. Let $z_{1}=e^{a_{1}} e^{b_{1} i}$ and $z_{2}=e^{a_{2}} e^{b_{2} i}$.

$$
z=\log _{z_{1}}\left(z_{2}\right) \Longleftrightarrow\left(z_{1}\right)^{z}=z_{2}, \text { thus } a_{2}=e^{a}\left(a_{1} \cos b-b_{1} \sin b\right) \text { and } b_{2}=e^{a}\left(b_{1} \cos b+a_{1} \sin b\right)
$$

$$
\begin{aligned}
a_{2}{ }^{2}+b_{2}{ }^{2} & =e^{2 a}\left(a_{1} \cos b-b_{1} \sin b\right)^{2}+e^{2 a}\left(b_{1} \cos b+a_{1} \sin b\right)^{2} \\
& =e^{2 a}\left(a_{1}{ }^{2} \cos ^{2} b-a_{1} b_{1} \cos b \sin b+b_{1}^{2} \sin ^{2} b+b_{1}{ }^{2} \cos ^{2} b+a_{1} b_{1} \cos b \sin b+a_{1}^{2} \sin ^{2} b\right) \\
& =e^{2 a}\left(a_{1}{ }^{2} \cos ^{2} b+a_{1}{ }^{2} \sin ^{2} b+b_{1}{ }^{2} \cos ^{2} b+b_{1}{ }^{2} \sin ^{2} b\right) \\
& =e^{2 a}\left(a_{1}{ }^{2}+b_{1}{ }^{2}\right) \\
a=\frac{1}{2} \ln \left(\frac{a_{2}{ }^{2}+b_{2}{ }^{2}}{a_{1}{ }^{2}+b_{1}{ }^{2}}\right) & \\
\frac{a_{2}}{b_{2}} & =\frac{e^{a}\left(a_{1} \cos b-b_{1} \sin b\right)}{e^{a}\left(b_{1} \cos b+a_{1} \sin b\right)} \\
a_{2}\left(b_{1} \cos b+a_{1} \sin b\right) & =b_{2}\left(a_{1} \cos b-b_{1} \sin b\right) \\
\cos b\left(a_{2} b_{1}-a_{1} b_{2}\right) & =-\sin b\left(a_{1} a_{2}+b_{1} b_{2}\right) \\
\sin b & =\frac{a_{1} b_{2}-a_{2} b_{1}}{a_{1} a_{2}+b_{1} b_{2}} \\
\cos b & =\operatorname{Atan}\left(\frac{a_{1} b_{2}-a_{2} b_{1}}{a_{1} a_{2}+b_{1} b_{2}}\right)
\end{aligned}
$$

Proof. Alternate proof of logarithm formula

$$
\begin{aligned}
z & =\log _{z_{1}}\left(z_{2}\right)=\frac{\ln z_{2}}{\ln z_{1}}=\frac{a_{2}+b_{2} i}{a_{1}+b_{1} i} \\
& =\frac{\left(a_{2}+b_{2} i\right)\left(a_{1}-b_{1} i\right)}{\left(a_{1}+b_{1} i\right)\left(a_{1}-b_{1} i\right)} \\
& =\frac{a_{1} a_{2}+b_{1} b_{2}+a_{1} b_{2} i-a_{2} b_{1} i}{a_{1}^{2}+b_{1}^{2}} \\
& =\frac{a_{1} a_{2}+b_{1} b_{2}}{a_{1}^{2}+b_{1}^{2}}+\frac{a_{1} b_{2}-a_{2} b_{1}}{a_{1}^{2}+b_{1}^{2}} i
\end{aligned}
$$

The result is in algebraic form and needs to be converted into complete form using conversion formula 2.4 .

$$
\begin{aligned}
a & =\frac{1}{2} \ln \frac{\left(a_{1} a_{2}+b_{1} b_{2}\right)^{2}+\left(a_{1} b_{2}-a_{2} b_{1}\right)^{2}}{\left(a_{1}^{2}+b_{1}{ }^{2}\right)^{2}} \\
& =\frac{1}{2} \ln \frac{a_{1}{ }^{2} a_{2}{ }^{2}+2 a_{1} a_{2} b_{1} b_{2}+b_{1}{ }^{2} b_{2}{ }^{2}+a_{1}{ }^{2} b_{2}{ }^{2}-2 a_{1} a_{2} b_{1} b_{2}+a_{2}{ }^{2} b_{1}{ }^{2}}{\left(a_{1}{ }^{2}+b_{1}{ }^{2}\right)^{2}} \\
& =\frac{1}{2} \ln \frac{a_{1}{ }^{2} a_{2}{ }^{2}+b_{1}{ }^{2} b_{2}{ }^{2}+a_{1}{ }^{2} b_{2}{ }^{2}+a_{2}{ }^{2} b_{1}{ }^{2}}{\left(a_{1}{ }^{2}+b_{1}{ }^{2}\right)^{2}}
\end{aligned}
$$




$$
\begin{aligned}
& =\frac{1}{2} \ln \frac{a_{1}^{2}\left(a_{2}^{2}+b_{2}^{2}\right)+b_{1}^{2}\left(a_{2}^{2}+b_{2}^{2}\right)}{\left(a_{1}^{2}+b_{1}^{2}\right)^{2}} \\
& =\frac{1}{2} \ln \frac{\left(a_{2}^{2}+b_{2}^{2}\right)\left(a_{1}^{2}+b_{1}^{2}\right)}{\left(a_{1}^{2}+b_{1}^{2}\right)^{2}} \\
& =\frac{1}{2} \ln \left(\frac{a_{2}^{2}+b_{2}^{2}}{a_{1}^{2}+b_{1}^{2}}\right) \\
& b=\operatorname{Atan}\left(\frac{\frac{a_{1} b_{2}-a_{2} b_{1}}{a_{1}^{2}+b_{1}^{2}}}{\frac{a_{1} a_{2}+b_{1} b_{2}}{a_{1}^{2}+b_{1}^{2}}}\right)=\operatorname{Atan}\left(\frac{a_{1} b_{2}-a_{2} b_{1}}{a_{1} a_{2}+b_{1} b_{2}}\right)
\end{aligned}
$$

Proof. Addition and subtraction formulas

Both operands need to be converted into algebraic form using the formula 2.1, since no identity can be used directly in complete form. Let $z_{1}=e^{a_{1}} \cos b_{1}+e^{a_{1}} \sin b_{1} i$ and $z_{2}=e^{a_{2}} \cos b_{2}+e^{a_{2}} \sin b_{2} i$

$$
\begin{aligned}
z & =z_{1} \pm z_{2}=\left(e^{a_{1}} \cos b_{1}+e^{a_{1}} \sin b_{1} i\right) \pm\left(e^{a_{2}} \cos b_{2}+e^{a_{2}} \sin b_{2} i\right) \\
& =\left(e^{a_{1}} \cos b_{1} \pm e^{a_{2}} \cos b_{2}\right)+\left(e^{a_{1}} \sin b_{1} \pm e^{a_{2}} \sin b_{2}\right) i
\end{aligned}
$$

The result is in algebraic form and needs to be converted into complete form using conversion formula 2.4 .

$$
\begin{aligned}
a= & \frac{1}{2} \ln \left(\left(e^{a_{1}} \cos b_{1} \pm e^{a_{2}} \cos b_{2}\right)^{2}+\left(e^{a_{1}} \sin b_{1} \pm e^{a_{2}} \sin b_{2}\right)^{2}\right) \\
= & \frac{1}{2} \ln \left(e^{2 a_{1}} \cos ^{2} b_{1} \pm 2 e^{a_{1}} e^{a_{2}} \cos b_{1} \cos b_{2}+e^{2 a_{2}} \cos ^{2} b_{2}+e^{2 a_{1}} \sin ^{2} b_{1} \pm 2 e^{a_{1}} e^{a_{2}} \sin b_{1} \sin b_{2}+\right. \\
& \left.e^{2 a_{2}} \sin ^{2} b_{2}\right) \\
= & \frac{1}{2} \ln \left(e^{2 a_{1}}\left(\cos ^{2} b_{1}+\sin ^{2} b_{1}\right)+e^{2 a_{2}}\left(\cos ^{2} b_{2}+\sin ^{2} b_{2}\right) \pm 2 e^{a_{1}} e^{a_{2}}\left(\cos b_{1} \cos b_{2}+\sin b_{1} \sin b_{2}\right)\right) \\
= & \frac{1}{2} \ln \left(e^{2 a_{1}}+e^{2 a_{2}} \pm 2 e^{a_{1}+a_{2}} \cos \left(b_{1}-b_{2}\right)\right) \\
b= & \operatorname{Atan}\left(\frac{e^{a_{1}} \sin b_{1} \pm e^{a_{2}} \sin b_{2}}{e^{a_{1}} \cos b_{1} \pm e^{a_{2}} \cos b_{2}}\right)
\end{aligned}
$$

Theorem 1. Within a number system composed of the sets $\mathbb{C} \subset \mathbb{E}, \mathbb{E}$ precision is the highest possible precision level obtained as result of a multiplication, division or exponentiation operation

From formulas 3.1, 3.2 and 3.3 we can easily deduce the result of the imaginary argument is not bounded by any limit and will be situated anywhere in $b \in \mathbb{R}$.

\section{Remark.}

The operations can be defined as functions $f: \mathbb{E} \times \mathbb{E} \rightarrow \mathbb{E}$, giving exactly four single variable continuous functions : $z \mapsto w \cdot z ; z \mapsto w / z$; power function $z \mapsto z^{w}$; exponential function $z \mapsto w^{z}$.

The complex exponentiation operation is more subtle since the exponent gets truncated to $\mathbb{C}$ precision by the cosine and sine functions used in the formula 3.3. On the other 
hand, the base and result require $\mathbb{E}$ precision.

Multiplication and division operands and results are at maximum $\mathbb{E}$ precise, no truncation is performed by the formulas 3.1 and 3.2. One can notice even with $\mathbb{C}$ precise operands, the result may be $\mathbb{E}$ precise.

Theorem 2. Within a number system composed of the sets $\mathbb{C} \subset \mathbb{E}, \mathbb{C}$ precision is the highest possible precision level obtained as result of a logarithm, addition or subtraction operation

The formulas 3.4, 3.5 and 3.6 use the atan2 function in the imaginary part, thus the result will always be situated inside the interval $b \in]-\pi ; \pi]$, which is exactly the definition of the $\mathbb{C}$ precision. The domain of the corresponding functions is therefore $f: \mathbb{E} \times \mathbb{E} \rightarrow \mathbb{C}$.

\section{Remark.}

Exactly four single variable continuous functions can be obtained: $z \mapsto w+z ; z \mapsto w-z$; $\operatorname{logarithm}$ function $z \mapsto \log _{w} z$; logarithm base function $z \mapsto \log _{z} w$. The singularities induced by the values 0 and $e^{0} e^{0 i}=1$ are studied in a further section.

The complex logarithm operation requires mixed precision, both operands require the complete form which can therefore be at maximum $\mathbb{E}$ precise, but the result is always at maximum $\mathbb{C}$ precise.

The addition and subtraction are the only operations not requiring the complete form hence no $\mathbb{E}$ precision, operands exceeding the required precision are truncated to $\mathbb{C}$ precision by formulas 3.5 and 3.6 .

\section{Theorem 3. All binary complex operations defined in $\mathbb{E}$ are monovalued}

From the formulas 3.1 to 3.6, we can deduce that both the real and imaginary part will always give a single valued results, since no real multivalued function is used in the formulas.

\section{Remark.}

The Atan function as defined in this study is monovalued. An alternate definition with a multivalued result of periodicity $2 \pi$ is possible and would imply the logarithm, addition and subtraction are multivalued in $\mathbb{E}$. Though a matter of definition, the single valuation arctangent is far more consistent algebraically and also geometrically as it will be seen in further sections. The logarithm, addition and subtraction results are intrinsically limited to $\mathbb{C}$ precision, in the same way a function defined as $f: \mathbb{Z} \times \mathbb{Z} \rightarrow \mathbb{N}$ returns one positive integer, not all integers included in the same equivalence class.

\section{Exponentials and logarithms identities in $\mathbb{E}$}

Theorem 4. All exponentiation identities valid in $\mathbb{R}_{+}^{*}$ are valid in $\mathbb{E}^{*}$ 
The result is strictly identical on both sides of the identity when $z_{1}, z_{2}, z_{3} \in \mathbb{E}^{*}$

$$
\begin{aligned}
\left(z_{1} z_{2}\right)^{z_{3}} & =z_{1}^{z_{3}} z_{2}^{z_{3}} \\
\left(\frac{z_{1}}{z_{2}}\right)^{z_{3}} & =\frac{z_{1} z_{3}}{z_{2} z_{3}} \\
z_{1}{ }^{z_{2}} z_{1}^{z_{3}} & =z_{1}^{z_{2}+z_{3}} \\
\frac{z_{1}^{z_{2}}}{z_{1}^{z_{3}}} & =z_{1}^{z_{2}-z_{3}} \\
\left(z_{1}^{z_{2}}\right)^{z_{3}} & =z_{1}^{z_{2} z_{3}}
\end{aligned}
$$

Theorem 5. The product and quotient logarithm identities valid in $\mathbb{R}_{+}^{*}$ are valid in $\mathbb{E}^{*}$

The result is strictly identical on both sides of the identity when $z_{1}, z_{2}, z_{3} \in \mathbb{E}^{*}$ and $z_{1} \neq e^{0} e^{0 i}$

$$
\begin{aligned}
& \log _{z_{1}}\left(z_{2} z_{3}\right)=\log _{z_{1}}\left(z_{2}\right)+\log _{z_{1}}\left(z_{3}\right) \\
& \log _{z_{1}}\left(\frac{z_{2}}{z_{3}}\right)=\log _{z_{1}}\left(z_{2}\right)-\log _{z_{1}}\left(z_{3}\right)
\end{aligned}
$$

Theorem 6. The power and base substitution logarithm identities valid in $\mathbb{R}_{+}^{*}$ are valid in $\mathbb{E}^{*}$ only at $\mathbb{C}$ precision level

The result truncated to $\mathbb{C}$ precision is strictly identical on both sides of the identity when $z_{1}, z_{2}, z_{3}, z_{4} \in \mathbb{E}^{*}$ and $z_{1}, z_{4} \neq e^{0} e^{0 i}$. The final operations on each side of the identity return different levels of precision, the identity cannot be a strict equality.

$$
\begin{aligned}
\log _{z_{1}}\left(z_{2} z_{3}\right) & =\left|z_{3} \log _{z_{1}}\left(z_{2}\right)\right|_{\mathbb{C}} \\
\log _{z_{1}}\left(z_{2}\right) & =\left|\frac{\log _{z_{4}}\left(z_{1}\right)}{\log _{z_{4}}\left(z_{2}\right)}\right|_{\mathbb{C}}
\end{aligned}
$$

As demonstrated within the following proofs, the trivial cases of exponential and logarithm identity failures given in the introduction dissapear when both sides of the identity equation are calculated in $\mathbb{E}$, thus when the formulas 3.1 to 3.6 are used at every calculation step.

Proof. $\left(z_{1} z_{2}\right)^{z_{3}}=z_{1}^{z_{3}} z_{2}^{z_{3}}$ is valid for all $z_{1}, z_{2}, z_{3} \in \mathbb{E}^{*}$

Combining the multiplication and exponentiation formulas 3.1 and 3.3 , let $z_{1}=e^{a_{1}} e^{b_{1} i}, z_{2}=$ $e^{a_{2}} e^{b_{2} i}$ and $z_{3}=e^{a_{3}} e^{b_{3} i}$

$$
\begin{aligned}
z & =\left(z_{1} z_{2}\right)^{z_{3}} \\
a & =e^{a_{3}}\left(\left(a_{1}+a_{2}\right) \cos b_{3}-\left(b_{1}+b_{2}\right) \sin b_{3}\right) \\
b & =e^{a_{3}}\left(\left(b_{1}+b_{2}\right) \cos b_{3}+\left(a_{1}+a_{2}\right) \sin b_{3}\right) \\
z & =z_{1}^{z_{3}} z_{2}^{z_{3}} \\
a & =e^{a_{3}}\left(a_{1} \cos b_{3}-b_{1} \sin b_{3}\right)+e^{a_{3}}\left(a_{2} \cos b_{3}-b_{2} \sin b_{3}\right) \\
& =e^{a_{3}}\left(\left(a_{1}+a_{2}\right) \cos b_{3}-\left(b_{1}+b_{2}\right) \sin b_{3}\right) \\
b & =e^{a_{3}}\left(b_{1} \cos b_{3}+a_{1} \sin b_{3}\right)+e^{a_{3}}\left(b_{2} \cos b_{3}+a_{2} \sin b_{3}\right) \\
& =e^{a_{3}}\left(\left(b_{1}+b_{2}\right) \cos b_{3}+\left(a_{1}+a_{2}\right) \sin b_{3}\right)
\end{aligned}
$$


Example 3. $(-1 \cdot-1)^{\frac{1}{2}} \neq(-1)^{\frac{1}{2}} \cdot(-1)^{\frac{1}{2}}$

$$
\begin{aligned}
& (-1 \cdot-1)^{\frac{1}{2}} \Longrightarrow\left(e^{\pi i} e^{\pi i}\right)^{\frac{1}{2}}=\left(e^{2 \pi i}\right)^{\frac{1}{2}}=e^{\pi i}=-1 \\
& (-1)^{\frac{1}{2}} \cdot(-1)^{\frac{1}{2}} \Longrightarrow\left(e^{\pi i}\right)^{\frac{1}{2}}\left(e^{\pi i}\right)^{\frac{1}{2}}=e^{\frac{\pi}{2} i} e^{\frac{\pi}{2} i}=e^{\pi i}=-1
\end{aligned}
$$

When the first expression is evaluated in algebraic form in $\mathbb{C}$, the result is 1 , the reason of the dissimilarity is because the result of the multiplication $-1 \cdot-1$ was implicitly truncated to a $\mathbb{C}$ precision level. In $\mathbb{E}$ equating $-1 \cdot-1=1$ is an over simplification : $e^{\pi i} e^{\pi i}=e^{2 \pi i} \neq e^{0 i}$, though in algebraic form the 2 values are indistinctive. This imprecision, invisible at first glance, is revealed when the exponent $\frac{1}{2}$ is applied on $e^{2 \pi i}$ or $e^{0 i}$ giving different values, respectively -1 and 1 . Similarly, $-i \cdot-i=e^{-\frac{\pi}{2} i} e^{-\frac{\pi}{2} i}=e^{-\pi i} \neq e^{\pi i}$ and $-1 \cdot i=e^{\pi i} e^{\frac{\pi}{2} i}=e^{\frac{3 \pi}{2} i} \neq e^{-\frac{\pi}{2} i}$. On the other hand, $i \cdot i=-1$ and $i \cdot-i=1$ are always valid.

Proof. $\left(z_{1} / z_{2}\right)^{z_{3}}=z_{1}^{z_{3}} / z_{2}^{z_{3}}$ is valid for all $z_{1}, z_{2}, z_{3} \in \mathbb{E}^{*}$

Combining the division and exponentiation formulas 3.2 and 3.3

$$
\begin{aligned}
z & =\left(\frac{z_{1}}{z_{2}}\right)^{z_{3}} \\
a & =e^{a_{3}}\left(\left(a_{1}-a_{2}\right) \cos b_{3}-\left(b_{1}-b_{2}\right) \sin b_{3}\right) \\
b & =e^{a_{3}}\left(\left(b_{1}-b_{2}\right) \cos b_{3}+\left(a_{1}-a_{2}\right) \sin b_{3}\right) \\
z & =\frac{z_{1}^{z_{3}}}{z_{2}^{z_{3}}} \\
a & =e^{a_{3}}\left(a_{1} \cos b_{3}-b_{1} \sin b_{3}\right)-e^{a_{3}}\left(a_{2} \cos b_{3}-b_{2} \sin b_{3}\right) \\
& =e^{a_{3}}\left(\left(a_{1}-a_{2}\right) \cos b_{3}-\left(b_{1}-b_{2}\right) \sin b_{3}\right) \\
b & =e^{a_{3}}\left(b_{1} \cos b_{3}+a_{1} \sin b_{3}\right)-e^{a_{3}}\left(b_{2} \cos b_{3}+a_{2} \sin b_{3}\right) \\
& =e^{a_{3}}\left(\left(b_{1}-b_{2}\right) \cos b_{3}+\left(a_{1}-a_{2}\right) \sin b_{3}\right)
\end{aligned}
$$

Example 4. $(1 /-1)^{\frac{1}{2}} \neq(1)^{\frac{1}{2}} /(-1)^{\frac{1}{2}}$

$$
\begin{aligned}
& \left(\frac{1}{-1}\right)^{\frac{1}{2}} \Longrightarrow\left(\frac{e^{0 i}}{e^{\pi i}}\right)^{\frac{1}{2}}=\left(e^{-\pi i}\right)^{\frac{1}{2}}=e^{-\frac{\pi}{2} i}=-i \\
& \frac{(1)^{\frac{1}{2}}}{(-1)^{\frac{1}{2}}} \Longrightarrow \frac{\left(e^{0 i}\right)^{\frac{1}{2}}}{\left(e^{\pi i}\right)^{\frac{1}{2}}}=\frac{e^{0 i}}{e^{\frac{\pi}{2} i}}=e^{-\frac{\pi}{2} i}=-i
\end{aligned}
$$

When the first expression is evaluated in algebraic form, the result is i. The error here is to consider $1 /-1=-1$ which is an implicit truncation at $\mathbb{C}$ precision level. In $\mathbb{E} e^{0 i} / e^{\pi i}=e^{-\pi i} \neq e^{\pi i}$. The exponent $\frac{1}{2}$ applied on $e^{\pi i}$ or $e^{-\pi i}$ giving different values in $\mathbb{C}$, respectively $-i$ and $i$. Similarly, $-1 /-i=e^{\pi i} / e^{-\frac{\pi}{2} i}=e^{\frac{3 \pi}{2} i} \neq e^{-\frac{\pi}{2} i}$ and $-i / i=e^{-\frac{\pi}{2} i} / e^{\frac{\pi}{2} i}=e^{-\pi i} \neq e^{\pi i}$.

Proof. $\left(z_{1}^{z_{2}}\right)^{z_{3}}=z_{1}^{z_{2} z_{3}}$ is valid for all $z_{1}, z_{2}, z_{3} \in \mathbb{E}^{*}$

Combining the multiplication and exponentiation formulas 3.1 and 3.3

$$
\begin{aligned}
& z=z_{1}^{z_{2} z_{3}} \\
& a=e^{a_{2}+a_{3}}\left(a_{1} \cos \left(b_{2}+b_{3}\right)-b_{1} \sin \left(b_{2}+b_{3}\right)\right)
\end{aligned}
$$




$$
\begin{aligned}
b & =e^{a_{2}+a_{3}}\left(b_{1} \cos \left(b_{2}+b_{3}\right)+a_{1} \sin \left(b_{2}+b_{3}\right)\right) \\
z & =\left(z_{1}^{z_{2}}\right)^{z_{3}} \\
a & =e^{a_{3}}\left(e^{a_{2}}\left(a_{1} \cos b_{2}-b_{1} \sin b_{2}\right) \cos b_{3}-e^{a_{2}}\left(b_{1} \cos b_{2}+a_{1} \sin b_{2}\right) \sin b_{3}\right) \\
& =e^{a_{2}} e^{a_{3}}\left(a_{1} \cos b_{2} \cos b_{3}-b_{1} \sin b_{2} \cos b_{3}-b_{1} \cos b_{2} \sin b_{3}-a_{1} \sin b_{2} \sin b_{3}\right) \\
& =e^{a_{2}+a_{3}}\left(a_{1}\left(\cos b_{2} \cos b_{3}-\sin b_{2} \sin b_{3}\right)-b_{1}\left(\sin b_{2} \cos b_{3}+\cos b_{2} \sin b_{3}\right)\right. \\
& =e^{a_{2}+a_{3}}\left(a_{1} \cos \left(b_{2}+b_{3}\right)-b_{1} \sin \left(b_{2}+b_{3}\right)\right) \\
b & =e^{a_{3}}\left(e^{a_{2}}\left(b_{1} \cos b_{2}+a_{1} \sin b_{2}\right) \cos b_{3}+e^{a_{2}}\left(a_{1} \cos b_{2}-b_{1} \sin b_{2}\right) \sin b_{3}\right) \\
& =e^{a_{2}} e^{a_{3}}\left(b_{1} \cos b_{2} \cos b_{3}+a_{1} \sin b_{2} \cos b_{3}+a_{1} \cos b_{2} \sin b_{3}-b_{1} \sin b_{2} \sin b_{3}\right) \\
& =e^{a_{2}+a_{3}}\left(b_{1}\left(\cos b_{2} \cos b_{3}-\sin b_{2} \sin b_{3}\right)+a_{1}\left(\sin b_{2} \cos b_{3}+\cos b_{2} \sin b_{3}\right)\right. \\
& =e^{a_{2}+a_{3}}\left(b_{1} \cos \left(b_{2}+b_{3}\right)+a_{1} \sin \left(b_{2}+b_{3}\right)\right) \\
z & =\left(z_{1}^{z_{3}}\right)^{z_{2}} \\
a & =e^{a_{2}}\left(e^{a_{3}}\left(a_{1} \cos b_{3}-b_{1} \sin b_{3}\right) \cos b_{2}-e^{a_{3}}\left(b_{1} \cos b_{3}+a_{1} \sin b_{3}\right) \sin b_{2}\right) \\
& =e^{a_{2}+a_{3}}\left(a_{1} \cos \left(b_{2}+b_{3}\right)-b_{1} \sin \left(b_{2}+b_{3}\right)\right) \\
b & =e^{a_{2}}\left(e^{a_{3}}\left(b_{1} \cos b_{3}+a_{1} \sin b_{3}\right) \cos b_{2}+e^{a_{3}}\left(a_{1} \cos b_{3}-b_{1} \sin b_{3}\right) \sin b_{2}\right) \\
& =e^{a_{2}+a_{3}}\left(b_{1} \cos \left(b_{2}+b_{3}\right)+a_{1} \sin \left(b_{2}+b_{3}\right)\right)
\end{aligned}
$$

Example 5. $\left((i-1)^{2}\right)^{i} \neq(i-1)^{2 i}$

$$
\begin{aligned}
& \left((i-1)^{2}\right)^{i} \Longrightarrow\left(\left(e^{\frac{1}{2} \ln 2} e^{\frac{3 \pi}{4} i}\right)^{2}\right)^{i}=\left(e^{\ln 2} e^{\frac{3 \pi}{2} i}\right)^{i}=e^{i \ln 2} e^{-\frac{3 \pi}{2}} \\
& (i-1)^{2 i} \Longrightarrow\left(e^{\frac{1}{2} \ln 2} e^{\frac{3 \pi}{4}}\right)^{2 i}=e^{i \ln 2} e^{-\frac{3 \pi}{2}}
\end{aligned}
$$

When $(i-1)^{2}$ is evaluated in algebraic form, the result obtained is $(i-1)(i-1)=-2 i$ which is only true in $\mathbb{C}$. Some relevant precision for $\mathbb{E}$ has been lost during the evaluation $:(i-1)(i-1)=-2 i=e^{\ln 2} e^{-\frac{\pi}{2} i} \neq(i-1)^{2}=\left(e^{\frac{1}{2} \ln 2} e^{\frac{3 \pi}{4} i}\right)^{2}=e^{\ln 2} e^{\frac{3 \pi}{2} i}$.

Example 6. Clausen paradox [5] [8]

$$
\begin{aligned}
& \left(e^{1+2 \pi k i}\right)^{1+2 \pi k i}=e^{(1+2 \pi k i)^{2}}=e^{1+4 \pi k i-4 \pi^{2} k^{2}}=e^{1-4 \pi^{2} k^{2}} e^{4 \pi k i} \\
& \left(e^{1+2 \pi k i}\right)^{1+2 \pi k i}=e^{1+2 \pi k i}=e \neq e^{1-4 \pi^{2} k^{2}} e^{4 \pi k i} \text {, the equality holds only when } k=0 .
\end{aligned}
$$

In the first expression the exponentiation base is taken as multivalued $e^{1} e^{2 \pi k i}$, the exponent in algebraic form $1+2 \pi k i$ is also multivalued, with both $\mathrm{k}$ synchronised. Nothing wrong here. The result of the exponentiation will obviously be multivalued, the first formula given is correct. In the second expression no exponentiation is performed, instead a double truncation from $\mathbb{E}$ to $\mathbb{C}$ precision. Equating $e^{1+2 \pi k i}=e^{1} e^{2 \pi k i}=e \cdot 1=e$ is imprecise, $\left|e^{1+2 \pi k i}\right|_{\mathbb{C}}=e$ is correct. After the truncation only the value within the interval $b \in]-\pi ; \pi]$ remains thus when $k=0$.

Proof. $z_{1}^{z_{2}} z_{1}^{z_{3}}=z_{1}^{z_{2}+z_{3}}$ is valid for all $z_{1}, z_{2}, z_{3} \in \mathbb{E}^{*}$ 
The identity is similar to the identity $e^{w_{1}} e^{w_{2}}=e^{w_{1}+w_{2}}$, with $w_{1}, w_{2} \in \mathbb{C}$

$$
\begin{aligned}
z^{z_{1}+z_{2}} & =z^{z_{1}} z^{z_{2}} \\
\left(e^{a} e^{b i}\right)^{z_{1}+z_{2}} & =\left(e^{a} e^{b i}\right)^{z_{1}}\left(e^{a} e^{b i}\right)^{z_{2}} \\
\left(e^{a+b i}\right)^{z_{1}+z_{2}} & =\left(e^{a+b i}\right)^{z_{1}}\left(e^{a+b i}\right)^{z_{2}} \\
e^{(a+b i)\left(z_{1}+z_{2}\right)} & \left.=e^{(a+b i) z_{1}} e^{(a+b i) z_{2}} \quad \text { (all exponents can be reduced into the form } z=x+y i\right) \\
e^{(a+b i)\left(z_{1}+z_{2}\right)} & =e^{(a+b i) z_{1}+(a+b i) z_{2}} \\
e^{(a+b i)\left(z_{1}+z_{2}\right)} & =e^{(a+b i)\left(z_{1}+z_{2}\right)}
\end{aligned}
$$

The identity can be verified using the multiplication 3.1 and exponentiation 3.3 formulas and the conversion formulas 2.1 and 2.4. Let $z_{1}=e^{a_{1}} e^{b_{1} i}, z_{2}=e^{a_{2}} e^{b_{2} i}$ and $z_{3}=e^{a_{3}} e^{b_{3} i}$

$$
\begin{aligned}
& z=z_{1}^{z_{2}} z_{1}^{z_{3}} \\
& a=e^{a_{2}}\left(a_{1} \cos b_{2}-b_{1} \sin b_{2}\right)+e^{a_{3}}\left(a_{1} \cos b_{3}-b_{1} \sin b_{3}\right) \\
& b=e^{a_{2}}\left(b_{1} \cos b_{2}+a_{1} \sin b_{2}\right)+e^{a_{3}}\left(b_{1} \cos b_{3}+a_{1} \sin b_{3}\right) \\
& z=z_{1}^{z_{2}+z_{3}} \\
& =z_{1} e^{\left(a_{2} \cos b_{2}+e^{a_{2}} \sin b_{2} i\right)+\left(e^{a_{3}} \cos b_{3}+e^{a_{3}} \sin b_{3} i\right)} \\
& =z_{1}{ }^{\left(e^{a_{2}} \cos b_{2}+e^{a_{3}} \cos b_{3}\right)+\left(e^{a_{2}} \sin b_{2}+e^{a_{3}} \sin b_{3}\right) i} \\
& =z_{1}{ }^{c+d i} \\
& a=e^{\frac{1}{2} \ln \left(c^{2}+d^{2}\right)}\left(a_{1} \cos \left(\arctan \left(\frac{d}{c}\right)\right)-b_{1} \sin \left(\arctan \left(\frac{d}{c}\right)\right)\right) \\
& =\sqrt{c^{2}+d^{2}}\left(\frac{a_{1}}{\sqrt{1+\frac{d^{2}}{c^{2}}}}-\frac{\frac{b_{1} d}{c}}{\sqrt{1+\frac{d^{2}}{c^{2}}}}\right) \\
& =a_{1} c-b_{1} d \\
& =a_{1}\left(e^{a_{2}} \cos b_{2}+e^{a_{3}} \cos b_{3}\right)-b_{1}\left(e^{a_{2}} \sin b_{2}+e^{a_{3}} \sin b_{3}\right) \\
& =e^{a_{2}}\left(a_{1} \cos b_{2}-b_{1} \sin b_{2}\right)+e^{a_{3}}\left(a_{1} \cos b_{3}-b_{1} \sin b_{3}\right) \\
& b=e^{\frac{1}{2} \ln \left(c^{2}+d^{2}\right)}\left(b_{1} \cos \left(\arctan \left(\frac{d}{c}\right)\right)+a_{1} \sin \left(\arctan \left(\frac{d}{c}\right)\right)\right) \\
& =\sqrt{c^{2}+d^{2}}\left(\frac{b_{1}}{\sqrt{1+\frac{d^{2}}{c^{2}}}}+\frac{\frac{a_{1} d}{c}}{\sqrt{1+\frac{d^{2}}{c^{2}}}}\right) \\
& =b_{1} c+a_{1} d \\
& =b_{1}\left(e^{a_{2}} \cos b_{2}+e^{a_{3}} \cos b_{3}\right)+a_{1}\left(e^{a_{2}} \sin b_{2}+e^{a_{3}} \sin b_{3}\right) \\
& =e^{a_{2}}\left(b_{1} \cos b_{2}+a_{1} \sin b_{2}\right)+e^{a_{3}}\left(b_{1} \cos b_{3}+a_{1} \sin b_{3}\right)
\end{aligned}
$$

Proof. $z_{1}^{z_{2}} / z_{1}^{z_{3}}=z_{1}{ }^{z_{2}-z_{3}}$ is valid for all $z_{1}, z_{2}, z_{3} \in \mathbb{E}^{*}$

The identity is similar to the identity $e^{w_{1}} / e^{w_{2}}=e^{w_{1}-w_{2}}$, with $w_{1}, w_{2} \in \mathbb{C}$

$$
\begin{aligned}
z^{z_{1}-z_{2}} & =\frac{z^{z_{1}}}{z^{z_{2}}} \\
\left(e^{a} e^{b i}\right)^{z_{1}-z_{2}} & =\frac{\left(e^{a} e^{b i}\right)^{z_{1}}}{\left(e^{a} e^{b i}\right)^{z_{2}}}
\end{aligned}
$$




$$
\left(e^{a+b i}\right)^{z_{1}-z_{2}}=\frac{\left(e^{a+b i}\right)^{z_{1}}}{\left(e^{a+b i}\right)^{z_{2}}}
$$

$e^{(a+b i)\left(z_{1}-z_{2}\right)}=\frac{e^{(a+b i) z_{1}}}{e^{(a+b i) z_{2}}}$ (all exponents can be reduced into the form $\left.z=x+y i\right)$

$e^{(a+b i)\left(z_{1}-z_{2}\right)}=e^{(a+b i) z_{1}-(a+b i) z_{2}}$

$e^{(a+b i)\left(z_{1}-z_{2}\right)}=e^{(a+b i)\left(z_{1}-z_{2}\right)}$

Proof. $\log _{z_{1}}\left(z_{2}{ }^{z_{3}}\right)=z_{3} \log _{z_{1}}\left(z_{2}\right)$ is valid at $\mathbb{C}$ precision level for all $z_{1}, z_{2}, z_{3} \in \mathbb{E}^{*}$

Combining the multiplication and logarithm formulas 3.1 and 3.4

$$
\begin{aligned}
& z=z_{3} \log _{z_{1}}\left(z_{2}\right) \\
& a=a_{3}+\frac{1}{2} \ln \left(\frac{a_{2}^{2}+b_{2}^{2}}{a_{1}^{2}+b_{1}^{2}}\right) \\
& b=b_{3}+\operatorname{Atan}\left(\frac{a_{1} b_{2}-a_{2} b_{1}}{a_{1} a_{2}+b_{1} b_{2}}\right) i
\end{aligned}
$$

Combining the exponentiation and logarithm formulas 3.3 and 3.4

$$
\begin{aligned}
& z=\log _{z_{1}}\left(z_{2}^{z_{3}}\right) \\
& a=\frac{1}{2} \ln \left(\frac{e^{2 a_{3}}\left(a_{2} \cos b_{3}-b_{2} \sin b_{3}\right)^{2}+e^{2 a_{3}}\left(b_{2} \cos b_{3}+a_{2} \sin b_{3}\right)^{2}}{a_{1}{ }^{2}+b_{1}{ }^{2}}\right) \\
& =\frac{1}{2} \ln \left(\frac{e^{2 a_{3}}\left(a_{2}{ }^{2} \cos ^{2} b_{3}+b_{2}{ }^{2} \sin ^{2} b_{3}+b_{2}{ }^{2} \cos ^{2} b_{3}+b_{2}{ }^{2} \sin ^{2} b_{3} \pm 2 a_{2} b_{2} \cos b_{3} \sin b_{3}\right)}{a_{1}{ }^{2}+b_{1}{ }^{2}}\right) \\
& =\frac{1}{2} \ln \left(e^{2 a_{3}} \cdot \frac{\left(a_{2}^{2}+b_{2}^{2}\right)\left(\cos ^{2} b_{3}+\sin ^{2} b_{3}\right)}{a_{1}^{2}+b_{1}^{2}}\right) \\
& =a_{3}+\frac{1}{2} \ln \left(\frac{a_{2}^{2}+b_{2}^{2}}{a_{1}^{2}+b_{1}^{2}}\right) \\
& b=\operatorname{Atan}\left(\frac{a_{1} e^{a_{3}}\left(b_{2} \cos b_{3}+a_{2} \sin b_{3}\right)-e^{a_{3}}\left(a_{2} \cos b_{3}-b_{2} \sin b_{3}\right) b_{1}}{a_{1} e^{a_{3}}\left(a_{2} \cos b_{3}-b_{2} \sin b_{3}\right)+b_{1} e^{a_{3}}\left(b_{2} \cos b_{3}+a_{2} \sin b_{3}\right)}\right) \\
& =\operatorname{Atan}\left(\frac{a_{1} b_{2} \cos b_{3}+a_{1} a_{2} \sin b_{3}-a_{2} b_{1} \cos b_{3}+b_{1} b_{2} \sin b_{3}}{a_{1} a_{2} \cos b_{3}-a_{1} b_{2} \sin b_{3}+b_{1} b_{2} \cos b_{3}+a_{2} b_{1} \sin b_{3}}\right) \\
& =\operatorname{Atan}\left(\frac{\left(a_{1} b_{2}-a_{2} b_{1}\right) \cos b_{3}+\left(a_{1} a_{2}+b_{1} b_{2}\right) \sin b_{3}}{\left(a_{1} a_{2}+b_{1} b_{2}\right) \cos b_{3}-\left(a_{1} b_{2}-a_{2} b_{1}\right) \sin b_{3}}\right) \\
& =\operatorname{Atan}\left(\frac{\frac{a_{1} b_{2}-a_{2} b_{1}}{a_{1} a_{2}+b_{1} b_{2}}+\frac{\sin b_{3}}{\cos b_{3}}}{1-\frac{a_{1} b_{2}-a_{2} b_{1}}{a_{1} a_{2}+b_{1} b_{2}} \cdot \frac{\sin b_{3}}{\cos b_{3}}}\right) \\
& =\operatorname{Atan}\left(\frac{\sin b_{3}}{\cos b_{3}}\right)+\operatorname{Atan}\left(\frac{a_{1} b_{2}-a_{2} b_{1}}{a_{1} a_{2}+b_{1} b_{2}}\right) \\
& =\left|b_{3}\right|_{\mathbb{C}}+\operatorname{Atan}\left(\frac{a_{1} b_{2}-a_{2} b_{1}}{a_{1} a_{2}+b_{1} b_{2}}\right)
\end{aligned}
$$

Example 7. $\ln \left((-i)^{2}\right) \neq 2 \ln (-i)$

$\ln \left((-i)^{2}\right) \Longrightarrow \ln \left(\left(e^{-\frac{\pi i}{2}}\right)^{2}\right)=\ln \left(e^{-\pi i}\right)=-\pi i$ 
$2 \ln (-i) \Longrightarrow 2 \ln \left(e^{-\frac{\pi i}{2}}\right)=2 \cdot\left(-\frac{\pi i}{2}\right)=-\pi i$

Since $-i \cdot-i=e^{-\frac{\pi}{2} i} e^{-\frac{\pi}{2} i}=e^{-\pi i} \neq e^{\pi i}$

Example 8. $\log _{-2}\left((-2)^{5}\right) \neq 5 \log _{-2}(-2)$

In the following calculation done in $\mathbb{C}$ the exponentiation is not applied to the imaginary part, giving a wrong result. There is anyway no place in $\mathbb{C}$ to hold the exact result of $(-2)^{5}$

$\log _{-2}\left((-2)^{5}\right)=\log _{-2}(-32)=\frac{\ln (-32)}{\ln (-2)}=\frac{\ln (32)+\pi i}{\ln (2)+\pi i}=\frac{(\ln (32)+\pi i)(\ln (2)-\pi i)}{(\ln (2)+\pi i)(\ln (2)-\pi i)}$ $=\frac{\ln (32) \ln (2)+(\ln (32)-\ln (2)) \pi i+\pi^{2}}{(\ln (2))^{2}+\pi^{2}}=1.18568 \ldots+0.84157 \ldots i$

The same calculation in $\mathbb{E}$

$z_{1}=-2 \Longrightarrow e^{\ln 2} e^{\pi i}$

$z_{2}=(-2)^{5} \Longrightarrow\left(e^{\ln 2} e^{\pi i}\right)^{5}=e^{5 \ln 2} e^{5 \pi i}$

$z=\log _{z_{1}} z_{2}$

$a=\frac{1}{2} \ln \left(\frac{a_{2}{ }^{2}+b_{2}{ }^{2}}{a_{1}{ }^{2}+b_{1}{ }^{2}}\right)=\frac{1}{2} \ln \left(\frac{(5 \ln 2)^{2}+(5 \pi)^{2}}{(\ln 2)^{2}+\pi^{2}}\right)=\frac{1}{2} \ln \left(5^{2}\right)=\ln 5$

$b=\operatorname{Atan}\left(\frac{a_{1} b_{2}-b_{1} a_{2}}{a_{1} a_{2}+b_{1} b_{2}}\right)=\operatorname{Atan}\left(\frac{5 \pi \ln 2-5 \pi \ln 2}{2(\ln 2)^{2}+5 \pi^{2}}\right)=\operatorname{Atan}(0)=0$

$z=e^{\ln 5} e^{0 i}=5$

Example 9. Identity failure at $\mathbb{E}$ precision level

$\ln \left(\left(e^{\pi i}\right)^{e^{32 \pi i}}\right)=\ln \left(e^{\pi i}\right)=\pi i=e^{\frac{1}{2} \ln \left(\pi^{2}\right)} e^{\frac{\pi}{2} i}$

$e^{32 \pi i} \ln \left(e^{\pi i}\right)=e^{32 \pi i} e^{\frac{1}{2} \ln \left(\pi^{2}\right)} e^{\frac{\pi}{2} i}=e^{\frac{1}{2} \ln \left(\pi^{2}\right)} e^{\frac{65 \pi}{2} i}$

Proof. $\log _{z_{1}}\left(z_{2}\right)=\log _{z_{3}}\left(z_{2}\right) / \log _{z_{3}}\left(z_{1}\right)$ is valid at $\mathbb{C}$ precision for all $z_{1}, z_{2}, z_{3} \in \mathbb{E}^{*}$

$$
\begin{aligned}
& z=\log _{z_{1}}\left(z_{2}\right) \\
& a=\frac{1}{2} \ln \left(\frac{a_{2}^{2}+b_{2}^{2}}{a_{1}^{2}+b_{1}{ }^{2}}\right) \\
& b=\operatorname{Atan}\left(\frac{a_{1} b_{2}-a_{2} b_{1}}{a_{1} a_{2}+b_{1} b_{2}}\right)
\end{aligned}
$$

Combining the logarithm and division formulas 3.4 and 3.2

$$
\begin{aligned}
z & =\frac{\log _{z_{3}}\left(z_{2}\right)}{\log _{z_{3}}\left(z_{1}\right)} \\
a & =\frac{1}{2} \ln \left(\frac{a_{2}{ }^{2}+b_{2}{ }^{2}}{a_{3}{ }^{2}+b_{3}{ }^{2}}\right)-\frac{1}{2} \ln \left(\frac{{a_{1}}^{2}+b_{1}{ }^{2}}{a_{3}{ }^{2}+b_{3}{ }^{2}}\right) \\
& =\frac{1}{2}\left(\ln \left({a_{2}}^{2}+{b_{2}}^{2}\right)-\ln \left({a_{3}}^{2}+b_{3}{ }^{2}\right)-\ln \left({a_{1}}^{2}+b_{1}{ }^{2}\right)+\ln \left(a_{3}{ }^{2}+b_{3}{ }^{2}\right)\right)
\end{aligned}
$$




$$
\begin{aligned}
& =\frac{1}{2}\left(\ln \left(a_{2}^{2}+b_{2}^{2}\right)-\ln \left(a_{1}^{2}+b_{1}^{2}\right)\right) \\
& =\frac{1}{2} \ln \left(\frac{a_{2}{ }^{2}+b_{2}^{2}}{a_{1}^{2}+b_{1}{ }^{2}}\right) \\
b & =\operatorname{Atan}\left(\frac{a_{3} b_{2}-a_{2} b_{3}}{a_{3} a_{2}+b_{3} b_{2}}\right)-\operatorname{Atan}\left(\frac{a_{3} b_{1}-a_{1} b_{3}}{a_{3} a_{1}+b_{3} b_{1}}\right) \\
& =\operatorname{Atan}\left(\frac{\frac{a_{3} b_{2}-a_{2} b_{3}}{a_{3} a_{2}+b_{3} b_{2}}-\frac{a_{3} b_{1}-a_{1} b_{3}}{a_{3} a_{1}+b_{3} b_{1}}}{1+\frac{a_{3} b_{2}-a_{2} b_{3}}{a_{3} a_{2}+b_{3} b_{2}} \cdot \frac{a_{3} b_{1}-a_{1} b_{3}}{a_{3} b_{1} b_{1}}}\right) \\
& =\operatorname{Atan}\left(\frac{\left(a_{3} b_{2}-a_{2} b_{3}\right)\left(a_{3} a_{1}+b_{3} b_{1}\right)-\left(a_{3} b_{1}-a_{1} b_{3}\right)\left(a_{3} a_{2}+b_{3} b_{2}\right)}{\left(a_{3} a_{1}+b_{3} b_{1}\right)\left(a_{3} a_{2}+b_{3} b_{2}\right)+\left(a_{3} b_{2}-a_{2} b_{3}\right)\left(a_{3} b_{1}-b_{3} a_{1}\right)}\right) \\
& =\operatorname{Atan}\left(\frac{a_{1} a_{3}{ }^{2} b_{2}+a_{3} b_{1} b_{2} b_{3}-a_{1} a_{2} a_{3} b_{3}-a_{2} b_{1} b_{3}{ }^{2}-a_{2} a_{3}{ }^{2} b_{1}-a_{3} b_{1} b_{2} b_{3}+a_{1} a_{2} a_{3} b_{3}+a_{1} b_{2} b_{3}{ }^{2}}{a_{1} a_{2} a_{3}{ }^{2}+a_{1} a_{3} b_{2} b_{3}+a_{2} a_{3} b_{1} b_{3}+b_{1} b_{2} b_{3}{ }^{2}+a_{3}{ }^{2} b_{1} b_{2}-a_{1} a_{3} b_{2} b_{3}-a_{2} a_{3} b_{1} b_{3}+a_{1} a_{2} b_{3}{ }^{2}}\right) \\
& =\operatorname{Atan}\left(\frac{\left(a_{3}{ }^{2}+b_{3}{ }^{2}\right)\left(a_{1} b_{2}-a_{2} b_{1}\right)}{\left(a_{3}{ }^{2}+b_{3}{ }^{2}\right)\left(a_{1} a_{2}+b_{1} b_{2}\right)}\right) \\
& =\operatorname{Atan}\left(\frac{a_{1} b_{2}-a_{2} b_{1}}{a_{1} a_{2}+b_{1} b_{2}}\right)
\end{aligned}
$$

Example 10. Identity failure at $\mathbb{E}$ precision level

$$
\log _{\frac{1}{4}}(4)=-1=e^{\pi i} \quad \frac{\log _{2}(4)}{\log _{2}\left(\frac{1}{4}\right)}=\frac{2}{-2}=\frac{e^{\ln 2} e^{0 i}}{e^{\ln 2} e^{\pi i}}=e^{-\pi i}
$$

Proof. $\log _{z_{1}}\left(z_{2} z_{3}\right)=\log _{z_{1}} z_{2}+\log _{z_{1}} z_{3}$ is valid for all $z_{1}, z_{2}, z_{3} \in \mathbb{E}^{*}$

Combining the multiplication and logarithm formulas 3.1 and 3.4

$$
\begin{aligned}
& z=\log _{z_{1}}\left(z_{2} z_{3}\right) \\
& a=\frac{1}{2} \ln \left(\frac{\left(a_{2}+a_{3}\right)^{2}+\left(b_{2}+b_{3}\right)^{2}}{a_{1}{ }^{2}+b_{1}{ }^{2}}\right) \\
& b=\operatorname{Atan}\left(\frac{a_{1}\left(b_{2}+b_{3}\right)-b_{1}\left(a_{2}+a_{3}\right)}{a_{1}\left(a_{2}+a_{3}\right)+b_{1}\left(b_{2}+b_{3}\right)}\right)
\end{aligned}
$$

For simplicity, the algebraic form is used in the following equation, since neither the logarithm nor the addition require the complete form for the result representation

$$
\begin{aligned}
z & =\log _{z_{1}}\left(z_{2}\right)+\log _{z_{1}}\left(z_{3}\right) \\
& =\frac{a_{1} a_{2}+b_{1} b_{2}}{a_{1}{ }^{2}+b_{1}{ }^{2}}+\frac{a_{1} b_{2}-a_{2} b_{1}}{a_{1}{ }^{2}+b_{1}{ }^{2}} i+\frac{a_{1} a_{3}+b_{1} b_{3}}{a_{1}{ }^{2}+b_{1}{ }^{2}}+\frac{a_{1} b_{3}-a_{3} b_{1}}{a_{1}{ }^{2}+b_{1}{ }^{2}} i \\
& =\frac{a_{1} a_{2}+b_{1} b_{2}+a_{1} a_{3}+b_{1} b_{3}}{a_{1}{ }^{2}+b_{1}{ }^{2}}+\frac{a_{1} b_{2}-a_{2} b_{1}+a_{1} b_{3}-a_{3} b_{1}}{a_{1}{ }^{2}+b_{1}{ }^{2}} i
\end{aligned}
$$

The result in algebraic form needs to be converted into complete form using conversion formula 2.4

$a=\frac{1}{2} \ln \left(\frac{\left(a_{1} a_{2}+b_{1} b_{2}+a_{1} a_{3}+b_{1} b_{3}\right)^{2}+\left(a_{1} b_{2}-a_{2} b_{1}+a_{1} b_{3}-a_{3} b_{1}\right)^{2}}{\left(a_{1}^{2}+b_{1}^{2}\right)^{2}}\right)$ 


$$
\begin{aligned}
& =\frac{1}{2} \ln \left(\frac{\left(a_{1}\left(a_{2}+a_{3}\right)+b_{1}\left(b_{2}+b_{3}\right)\right)^{2}+\left(a_{1}\left(b_{2}+b_{3}\right)-b_{1}\left(a_{2}+a_{3}\right)\right)^{2}}{\left(a_{1}^{2}+b_{1}^{2}\right)^{2}}\right) \\
& =\frac{1}{2} \ln \left(\frac{a_{1}^{2}\left(a_{2}+a_{3}\right)^{2}+b_{1}^{2}\left(b_{2}+b_{3}\right)^{2}+a_{1}^{2}\left(b_{2}+b_{3}\right)^{2}+b_{1}^{2}\left(a_{2}+a_{3}\right)^{2}}{\left(a_{1}^{2}+b_{1}^{2}\right)^{2}}\right) \\
& =\frac{1}{2} \ln \left(\frac{\left(a_{1}^{2}+b_{1}^{2}\right)\left(\left(a_{2}+a_{3}\right)^{2}+\left(b_{2}+b_{3}\right)^{2}\right)}{\left(a_{1}^{2}+b_{1}^{2}\right)^{2}}\right) \\
& =\frac{1}{2} \ln \left(\frac{\left.\left(a_{2}+a_{3}\right)^{2}+\left(b_{2}+b_{3}\right)^{2}\right)}{a_{1}^{2}+b_{1}^{2}}\right) \\
& b=\operatorname{Atan}\left(\frac{a_{1} b_{2}-a_{2} b_{1}+a_{1} b_{3}-a_{3} b_{1}}{a_{1} a_{2}+b_{1} b_{2}+a_{1} a_{3}+b_{1} b_{3}}\right) \\
& =\operatorname{Atan}\left(\frac{a_{1}\left(b_{2}+b_{3}\right)-b_{1}\left(a_{2}+a_{3}\right)}{a_{1}\left(a_{2}+a_{3}\right)+b_{1}\left(b_{2}+b_{3}\right)}\right)
\end{aligned}
$$

Example 11. $\ln (-1 \cdot-1) \neq \ln (-1)+\ln (-1)$

$\ln (-1 \cdot-1)=\ln \left(e^{\pi i} e^{\pi i}\right)=\ln \left(e^{2 \pi i}\right)=2 \pi i$

$\ln (-1)+\ln (-1)=\pi i+\pi i=2 \pi i$

Proof. $\log _{z_{1}}\left(z_{2} / z_{3}\right)=\log _{z_{1}} z_{2}-\log _{z_{1}} z_{3}$ is valid for all $\mathbf{z}_{1}, \mathrm{z}_{2}, \mathrm{z}_{3} \in \mathbb{E}^{*}$

Combining the division and logarithm formulas 3.2 and 3.4

$$
\begin{aligned}
& z=\log _{z_{1}}\left(\frac{z_{2}}{z_{3}}\right) \\
& a=\frac{1}{2} \ln \left(\frac{\left(a_{2}-a_{3}\right)^{2}+\left(b_{2}-b_{3}\right)^{2}}{a_{1}{ }^{2}+b_{1}{ }^{2}}\right) \\
& b=\operatorname{Atan}\left(\frac{a_{1}\left(b_{2}-b_{3}\right)-b_{1}\left(a_{2}-a_{3}\right)}{a_{1}\left(a_{2}-a_{3}\right)+b_{1}\left(b_{2}-b_{3}\right)}\right) \\
& z=\log _{z_{1}}\left(z_{2}\right)-\log _{z_{1}}\left(z_{3}\right) \\
& =\frac{a_{1} a_{2}+b_{1} b_{2}}{a_{1}{ }^{2}+b_{1}^{2}}+\frac{a_{1} b_{2}-a_{2} b_{1}}{a_{1}^{2}+b_{1}^{2}} i-\frac{a_{1} a_{3}+b_{1} b_{3}}{a_{1}^{2}+b_{1}^{2}}-\frac{a_{1} b_{3}-a_{3} b_{1}}{a_{1}^{2}+b_{1}{ }^{2}} i \\
& =\frac{a_{1} a_{2}+b_{1} b_{2}-a_{1} a_{3}-b_{1} b_{3}}{a_{1}^{2}+b_{1}^{2}}+\frac{a_{1} b_{2}-a_{2} b_{1}-a_{1} b_{3}+a_{3} b_{1}}{a_{1}^{2}+b_{1}{ }^{2}} i \\
& a=\frac{1}{2} \ln \left(\frac{\left(a_{1} a_{2}+b_{1} b_{2}-a_{1} a_{3}-b_{1} b_{3}\right)^{2}+\left(a_{1} b_{2}-a_{2} b_{1}-a_{1} b_{3}+a_{3} b_{1}\right)^{2}}{\left(a_{1}^{2}+b_{1}^{2}\right)^{2}}\right) \\
& =\frac{1}{2} \ln \left(\frac{\left(a_{1}\left(a_{2}-a_{3}\right)+b_{1}\left(b_{2}-b_{3}\right)\right)^{2}+\left(a_{1}\left(b_{2}-b_{3}\right)-b_{1}\left(a_{2}-a_{3}\right)\right)^{2}}{\left(a_{1}^{2}+b_{1}^{2}\right)^{2}}\right) \\
& =\frac{1}{2} \ln \left(\frac{a_{1}{ }^{2}\left(a_{2}-a_{3}\right)^{2}+b_{1}{ }^{2}\left(b_{2}-b_{3}\right)^{2}+a_{1}{ }^{2}\left(b_{2}-b_{3}\right)^{2}+b_{1}{ }^{2}\left(a_{2}-a_{3}\right)^{2}}{\left(a_{1}{ }^{2}+b_{1}{ }^{2}\right)^{2}}\right)
\end{aligned}
$$




$$
\begin{aligned}
& =\frac{1}{2} \ln \left(\frac{\left(a_{1}^{2}+b_{1}^{2}\right)\left(\left(a_{2}-a_{3}\right)^{2}+\left(b_{2}-b_{3}\right)^{2}\right)}{\left(a_{1}^{2}+b_{1}{ }^{2}\right)^{2}}\right) \\
& =\frac{1}{2} \ln \left(\frac{\left.\left(a_{2}-a_{3}\right)^{2}+\left(b_{2}-b_{3}\right)^{2}\right)}{a_{1}{ }^{2}+b_{1}{ }^{2}}\right) \\
& b=\operatorname{Atan}\left(\frac{a_{1} b_{2}-a_{2} b_{1}-a_{1} b_{3}+a_{3} b_{1}}{a_{1} a_{2}+b_{1} b_{2}-a_{1} a_{3}-b_{1} b_{3}}\right) \\
& =\operatorname{Atan}\left(\frac{a_{1}\left(b_{2}-b_{3}\right)-b_{1}\left(a_{2}-a_{3}\right)}{a_{1}\left(a_{2}-a_{3}\right)+b_{1}\left(b_{2}-b_{3}\right)}\right)
\end{aligned}
$$

\section{$5 \quad$ Formulas for transcendental equations}

The formulas 3.1 to 3.6 can be combined to obtain formulas linking the real and imaginary arguments of expressions using the complex operations.

Example 12. $z_{2}=z_{1}{ }^{w} \cdot w^{\alpha}$ where $w, z_{1}, z_{2} \in \mathbb{E}^{*}, z_{1} \neq e^{0} e^{0 i}, \alpha \in \mathbb{R}$

Explicit formulas linking the real and imaginary arguments $a_{w}, b_{w}$ of $w$ can be obtained.

$$
\begin{aligned}
& z_{2}=z_{1}{ }^{w} w^{\alpha} \\
& a_{2}=e^{a_{w}}\left(a_{1} \cos b_{w}-b_{1} \sin b_{w}\right)+a_{w} \alpha \\
& b_{2}=e^{a_{w}}\left(b_{1} \cos b_{w}+a_{1} \sin b_{w}\right)+b_{w} \alpha \\
& \left(a_{2}-a_{w} \alpha\right)^{2}=e^{2 a_{w}}\left(a_{1} \cos b_{w}-b_{1} \sin b_{w}\right)^{2} \\
& \left(b_{2}-b_{w} \alpha\right)^{2}=e^{2 a_{w}}\left(b_{1} \cos b_{w}+a_{1} \sin b_{w}\right)^{2} \\
& \left(a_{2}-a_{w} \alpha\right)^{2}+\left(b_{2}-b_{w} \alpha\right)^{2}=e^{2 a_{w}}\left(\left(a_{1} \cos b_{w}-b_{1} \sin b_{w}\right)^{2}+\left(b_{1} \cos b_{w}+a_{1} \sin b_{w}\right)^{2}\right) \\
& =e^{2 a_{w}}\left(a_{1}^{2} \cos ^{2} b_{w}+b_{1}^{2} \sin ^{2} b_{w}+b_{1}^{2} \cos ^{2} b_{w}+a_{1}^{2} \sin ^{2} b_{w} \pm 2 a_{1} b_{1} \cos b_{w} \sin b_{w}\right) \\
& =e^{2 a_{w}}\left(a_{1}^{2}\left(\cos ^{2} b_{w}+\sin ^{2} b_{w}\right)+b_{1}^{2}\left(\cos ^{2} b_{w}+\sin ^{2} b_{w}\right)\right) \\
& =e^{2 a_{w}}\left(a_{1}{ }^{2}+b_{1}{ }^{2}\right) \\
& \frac{b_{2}-b_{w} \alpha}{a_{2}-a_{w} \alpha}=\frac{a_{1} \cos b_{w}-b_{1} \sin b_{w}}{b_{1} \cos b_{w}+a_{1} \sin b_{w}}
\end{aligned}
$$

From which the final formulas are obtained :

$$
\begin{aligned}
e^{2 a_{w}}\left(a_{1}{ }^{2}+b_{1}{ }^{2}\right) & =\left(a_{2}-a_{w} \alpha\right)^{2}+\left(b_{2}-b_{w} \alpha\right)^{2} \\
b_{w}= & \frac{b_{2}-\sqrt{e^{2 a_{w}}\left(a_{1}{ }^{2}+b_{1}{ }^{2}\right)-\left(a_{2}-a_{w} \alpha\right)^{2}}}{\alpha} \\
a_{w}= & \frac{a_{2}-\frac{a_{1} \cos b_{w}-b_{1} \sin b_{w}}{b_{1} \cos b_{w}+a_{1} \sin b_{w}}\left(b_{2}-b_{w} \alpha\right)}{\alpha}
\end{aligned}
$$




$$
a_{w}=\ln \left(\frac{b_{2}-b_{w} \alpha}{b_{1} \cos b_{w}+a_{1} \sin b_{w}}\right)
$$

Example 13. $z_{2}=\log _{z_{1}}(w) \cdot w^{\alpha}$ where $w, z_{1}, z_{2} \in \mathbb{E}^{*}, w, z_{1} \neq e^{0} e^{0 i}, \alpha \in \mathbb{R}$

Explicit formulas linking the real and imaginary arguments $a_{w}, b_{w}$ of $w$ can be obtained.

$$
\begin{aligned}
& z_{2}=\log _{z_{1}}(w) w^{\alpha} \\
& a_{2}=\frac{1}{2} \ln \left(\frac{a_{w}{ }^{2}+b_{w}{ }^{2}}{a_{1}{ }^{2}+b_{1}{ }^{2}}\right)+a_{w} \alpha \\
& b_{2}=\operatorname{Atan}\left(\frac{a_{1} b_{w}-a_{w} b_{1}}{a_{1} a_{w}+b_{1} b_{w}}\right)+b_{w} \alpha=\operatorname{Atan}\left(\frac{b_{w}}{a_{w}}\right)-\operatorname{Atan}\left(\frac{b_{1}}{a_{1}}\right)+b_{w} \alpha
\end{aligned}
$$

From which the final formulas are obtained :

$$
\begin{aligned}
& b_{w}=\sqrt{e^{2\left(a_{2}-a_{w} \alpha\right)}\left(a_{1}{ }^{2}+b_{1}{ }^{2}\right)-a_{w}{ }^{2}} \\
& a_{w}=b_{w} \cot \left(b_{2}-b_{w} \alpha+\operatorname{Atan}\left(\frac{b_{1}}{a_{1}}\right)\right)
\end{aligned}
$$




\section{Geometric representation of $\mathbb{E}$}

\subsection{The complex helicoid}

The complex plane is clearly insufficient to represent $\mathbb{E}$ precise numbers, one can notice only $e^{a} e^{b i}$ with $\left.\left.b \in\right]-\pi ; \pi\right]$ can be positioned in a unique way. The lack of "space" is solved by an additional axis, hereafter named the i axis, on which the imaginary argument $\mathrm{b}$ can translate rectilinearly without any boundaries. The rotation of the imaginary argument $\mathrm{b}$ is maintained with a $2 \pi$ period, giving a unique perpendicular half straight line for each $\mathrm{b}$ argument on which the real part $e^{a}$ is positioned. Hereafter those half-lines are named "rays". Viewed in a three dimension euclidian space, with the origin situated at 0 on the $\mathrm{i}$ axis, every number $w=e^{a} e^{b i}$ can be given a unique orthogonal coordinate $(x, y, z)=\left(e^{a} \cos b, e^{a} \sin b, b\right)$. Thus the set $\mathbb{E}$ forms exactly an helicoid surface, herafter named the complex helicoid.

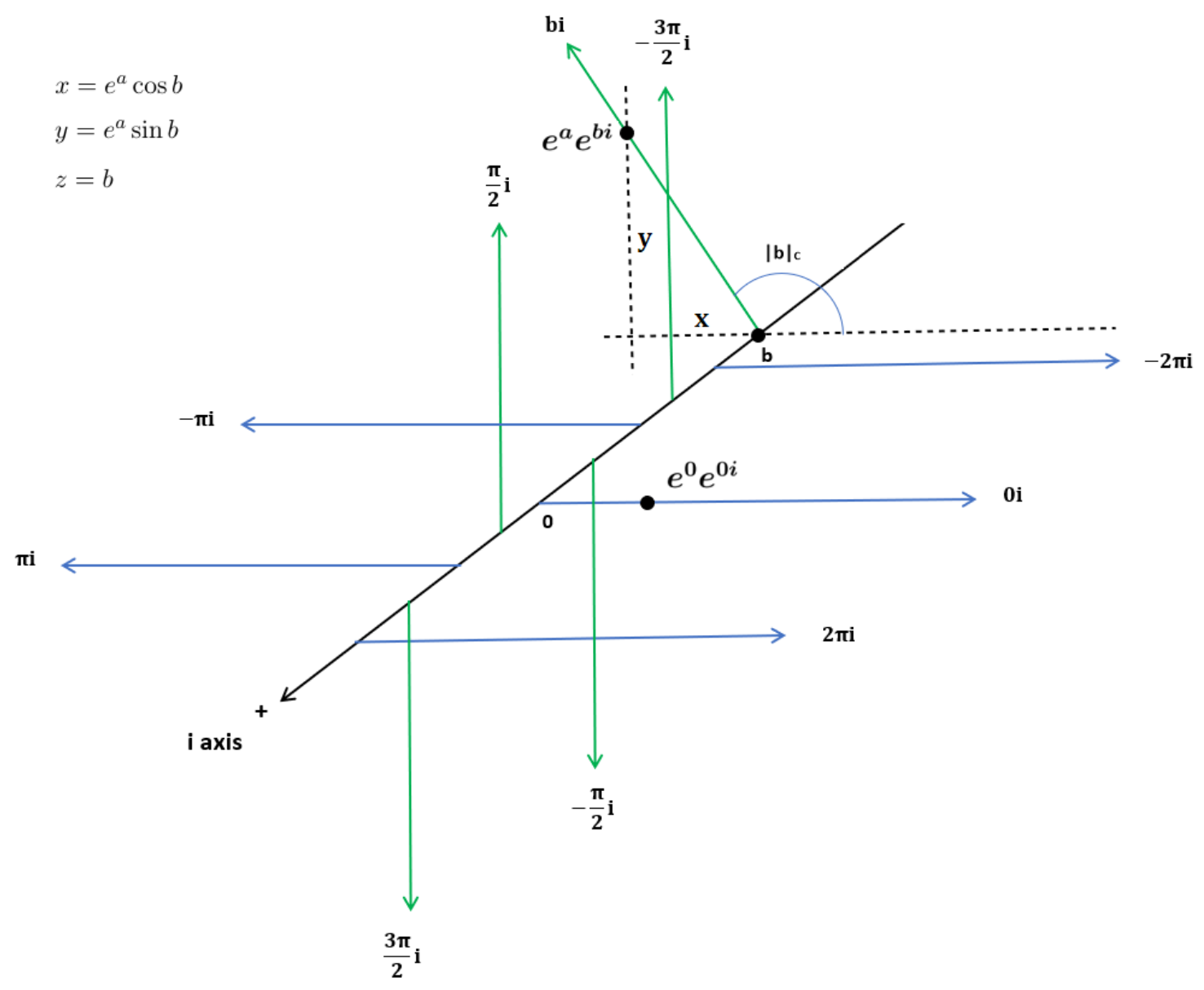

Figure 1: Representation of $e^{a} e^{b i}$ and $e^{0} e^{0 i}$ on the complex helicoid

The $\mathrm{i}$ axis is a singularity itself, on which only the value 0 can be positioned. The value 0 was included into the $\mathbb{E}$ set only for algebraic purpose. Zero and infinity are equally singularities without a unique positioning.

The complex helicoid is the counterpart of the complex plane for $\mathbb{C}$ and the real axis for $\mathbb{R}$. 


\subsection{Constant functions representation on the complex helicoid}

The constant function $w=e^{a}$ is the set of points situated at the position $e^{a}$ on each ray. The function appears as an infinite helix surrounding the $\mathrm{i}$ axis. The multiplication and division operations such as $w=e^{a \pm a^{\prime}}$ translate the position of the point on each ray, thus bring closer or further the helix to the $\mathrm{i}$ axis.

The constant function $w=e^{b i}$ is the set of points on a ray pointing in the direction given by $b$, excluding the 0 situated on the $i$ axis. The multiplication and division operations such as $w=e^{\left(b \pm b^{\prime}\right) i}$ operate a rotation and a translation around and along the $\mathrm{i}$ axis.

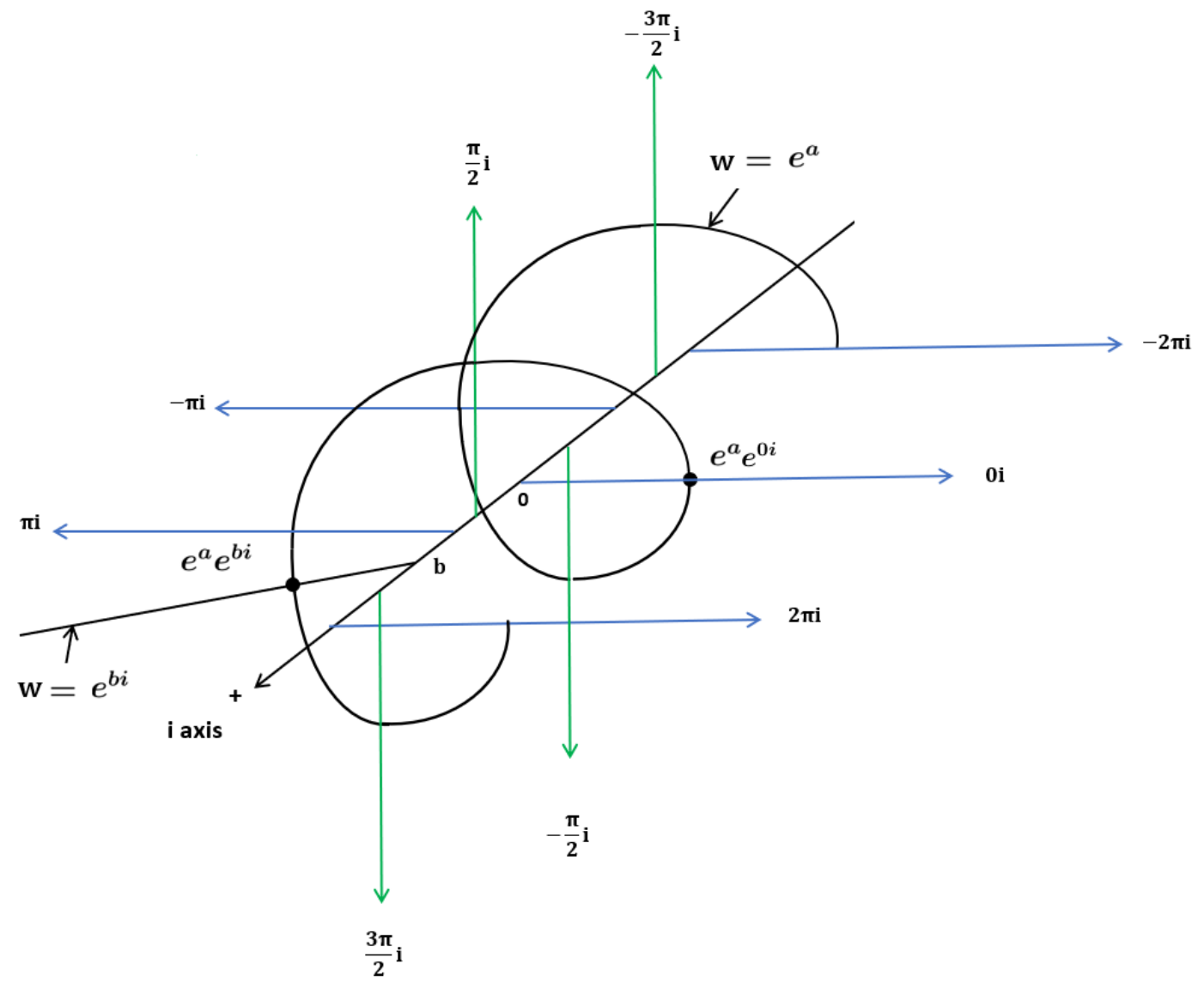

Figure 2: Constant functions $w=e^{a}$ and $w=e^{b i}$ representations on the complex helicoid 


\subsection{Complex helicoid projections on the plane}

The orthogonal projection of the complex helicoid $(x, y, z)$ to $(x, y, 0)$ represents the complex plane, through a new perspective. The projection corresponds exactly to a $\mathbb{C}$ truncation of $\mathbb{E}$ and can be noted as $P(w)=P\left(e^{a} e^{b i}\right)=P\left(e^{a} \cos b, e^{a} \sin b, b\right)=$ $\left(e^{a} \cos b, e^{a} \sin b, 0\right)$ or as a truncation $|w|_{\mathbb{C}}=\left|e^{a} e^{b i}\right|_{\mathbb{C}}=e^{a} \cos b+e^{a} \sin b i$. The singularity 0 is given the appearance of a normal point. The exponentials and logarithms identity failures in $\mathbb{C}$ represented on the complex plane are all due to a "careless" crossing of the Re- axis generating a $\mathbb{C}$ truncation. The projection should not be confused with the logarithmic representation of $\mathbb{E}$ which will be seen further, though both representations are graphicaly identical.

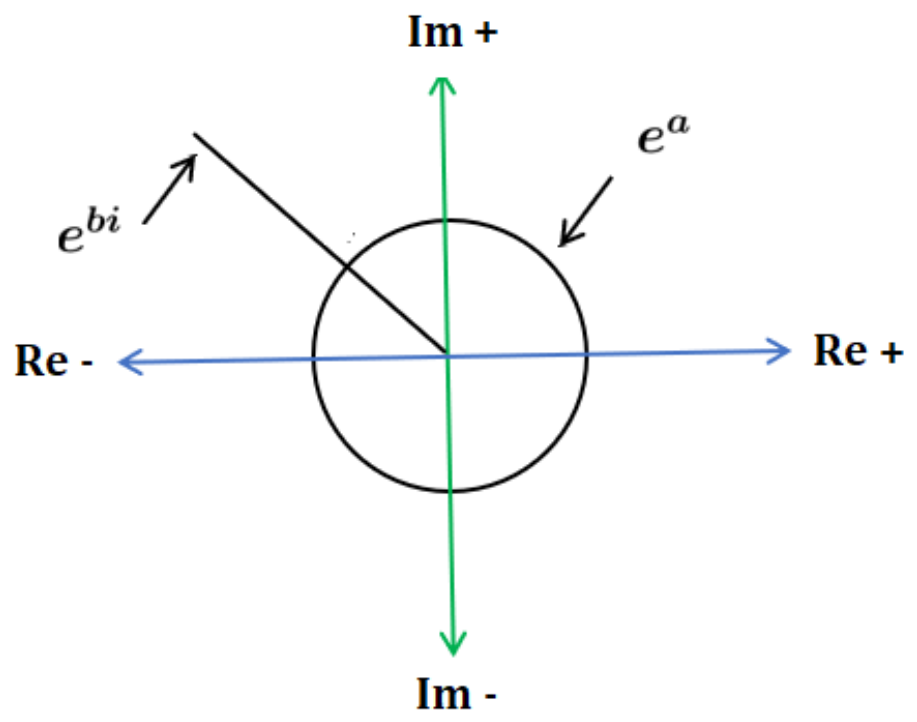

Figure 3: Projection of the complex helicoid $(x, y, z)$ to $(x, y, 0)$

Similarly, the orthogonal projections of the complex helicoid $(x, y, z)$ to $(x, 0, z)$ and $(x, y, z)$ to $(0, y, z)$, maps the constant helix into a cosine and sine curve. 


\subsection{Representation of the complex logarithm operation}

Let $z_{1}, z_{2} \in \mathbb{E}^{*}$ with $z_{1}, z_{2} \neq e^{0} e^{0 i}$ be 2 points on the complex helicoid. The representation of the point $z=\log _{z_{1}}\left(z_{2}\right)$ reveals, under a new perspective, a similar formula as the division on the complex plane.

$$
c_{1}=\sqrt{a_{1}^{2}+b_{1}^{2}} \quad c_{2}=\sqrt{a_{2}^{2}+b_{2}^{2}} \quad \alpha_{1}=\operatorname{Atan} \frac{b_{1}}{a_{1}} \quad \alpha_{2}=\operatorname{Atan} \frac{b_{2}}{a_{2}}
$$

Since $z=\log _{z_{1}}\left(z_{2}\right)=e^{\frac{1}{2} \ln \left(\frac{a_{2}^{2}+b_{2}^{2}}{a_{1}^{2}+b_{1}^{2}}\right)} e^{\operatorname{Atan}\left(\frac{a_{1} b_{2}-a_{2} b_{1}}{a_{1} a_{2}+b_{1} b_{2}}\right) i}=\frac{\sqrt{a_{2}^{2}+b_{2}^{2}}}{\sqrt{a_{1}^{2}+b_{1}^{2}}} e^{\left(\operatorname{Atan} \frac{b_{2}}{a_{2}}-\operatorname{Atan} \frac{b_{1}}{a_{1}}\right) i}$

$$
z=\log _{z_{1}}\left(z_{2}\right)=\frac{c_{2}}{c_{1}} e^{\left(\alpha_{2}-\alpha_{1}\right) i}
$$

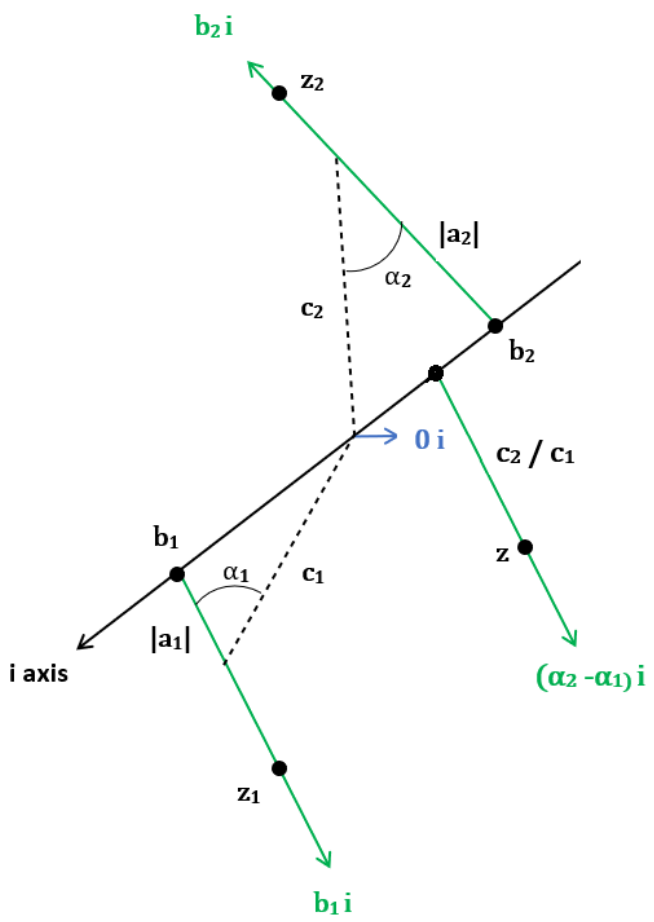

Figure 4: Logarithm operation representation on the complex helicoid 


\subsection{Representation of the complex exponentiation operation}

Let $z_{1}, z_{2} \in \mathbb{E}^{*}$ with $z_{1} \neq e^{0} e^{0 i}$ be 2 points on the complex helicoid. The representation of the point $z=z_{1}{ }^{z_{2}}$ is best visualised by 2 formulas. The exponent $z_{2}$ only being used at $\mathbb{C}$ precision, quite obviously the full $b_{2}$ distance on the $\mathrm{i}$ axis is not used in the formulas.

$$
x_{2}=e^{a_{2}} \cos b_{2} \quad y_{2}=e^{a_{2}} \sin b_{2} \quad c_{1}=\sqrt{a_{1}^{2}+b_{1}^{2}} \quad \alpha_{1}=\operatorname{Atan} \frac{b_{1}}{a_{1}} \quad \beta_{1}=\operatorname{Atan} \frac{a_{1}}{b_{1}}
$$

Since $z=z_{1}^{z_{2}}=e^{e^{a_{2}}\left(a_{1} \cos b_{2}-b_{1} \sin b_{2}\right)} e^{e^{a_{2}}\left(b_{1} \cos b_{2}+a_{1} \sin b_{2}\right) i}$

$$
z=z_{1}^{z_{2}}=e^{a_{1} x_{2}-b_{1} y_{2}} e^{\left(b_{1} x_{2}+a_{1} y_{2}\right) i}
$$

or $z=z_{1}^{z_{2}}=e^{e^{a_{2}} \sqrt{a_{1}^{2}+b_{1}^{2}} \cos \left(b_{2}+\operatorname{Atan} \frac{b_{1}}{a_{1}}\right)} e^{e^{a_{2}} \sqrt{a_{1}^{2}+b_{1}^{2}} \cos \left(b_{2}-\operatorname{Atan} \frac{a_{1}}{b_{1}}\right) i}$

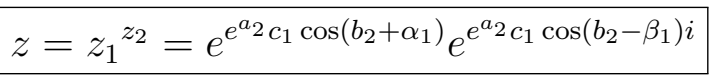

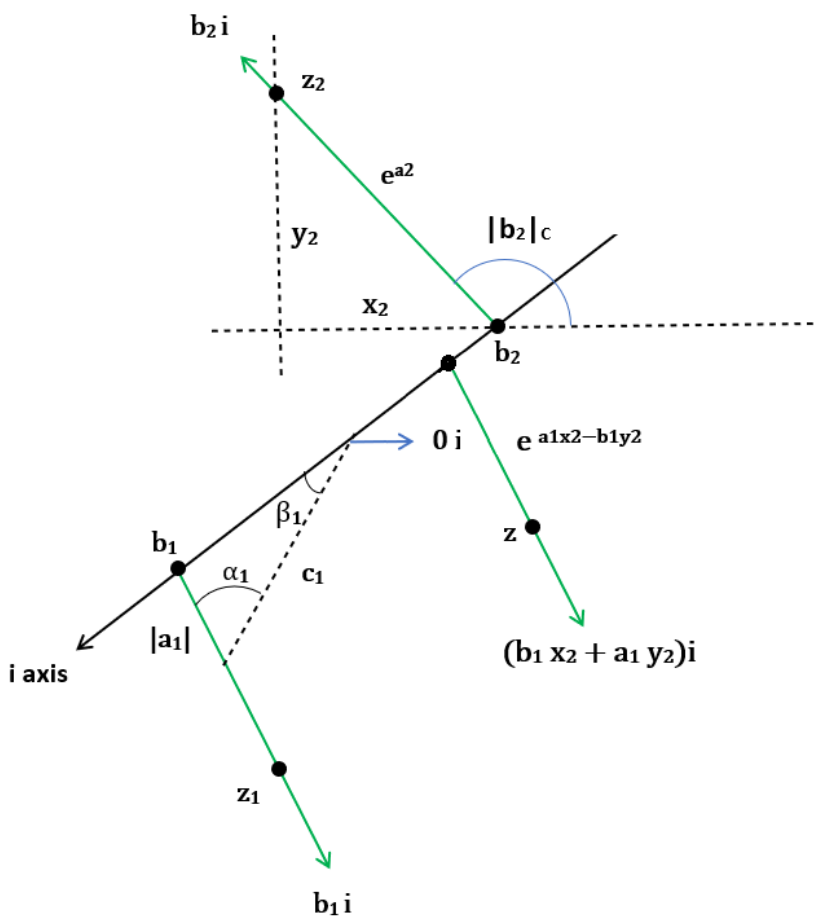

Figure 5: Exponentiation operation representation on the complex helicoid 


\subsection{Representation of the addition and subtraction operations}

The addition and subtraction do not require any $\mathbb{E}$ precision, representing them on the complex helicoid is basically useless, a projection on the complex plane is sufficient. Let $z_{1}, z_{2} \in \mathbb{E}^{*}$ by 2 points on the complex helicoid, with their corresponding projections $\left|z_{1}\right|_{\mathbb{C}}=x_{1}+y_{1} i$ and $\left|z_{2}\right|_{\mathbb{C}}=x_{2}+y_{2} i$ on the complex plane.

$x_{1}=e^{a_{1}} \cos b_{1} \quad y_{1}=e^{a_{1}} \sin b_{1} \quad x_{2}=e^{a_{2}} \cos b_{2} \quad y_{2}=e^{a_{2}} \sin b_{2}$

$z=z_{1} \pm z_{2}=e^{\frac{1}{2} \ln \left(e^{2 a_{1}}+e^{2 a_{2}} \pm 2 e^{a_{1}+a_{2}} \cos \left(b_{1}-b_{2}\right)\right)} e^{\operatorname{Atan}\left(\frac{e^{a_{1} \sin b_{1} \pm e^{a_{2}} \sin b_{2}}}{e^{a_{1}} \cos b_{1} \pm e^{a_{2}} \cos b_{2}}\right) i}$

$c=\sqrt{e^{2 a_{1}}+e^{2 a_{2}}-2 e^{a_{1}+a_{2}} \cos \left(b_{1}-b_{2}\right)} \quad d=\sqrt{e^{2 a_{1}}+e^{2 a_{2}}+2 e^{a_{1}+a_{2}} \cos \left(b_{1}-b_{2}\right)}$

$z=z_{1}-z_{2}=c \cdot e^{\operatorname{Atan}\left(\frac{y_{1}-y_{2}}{x_{1}-x_{2}}\right) i} \quad z=z_{1}+z_{2}=d \cdot e^{\operatorname{Atan}\left(\frac{y_{1}+y_{2}}{x_{1}+x_{2}}\right) i}$

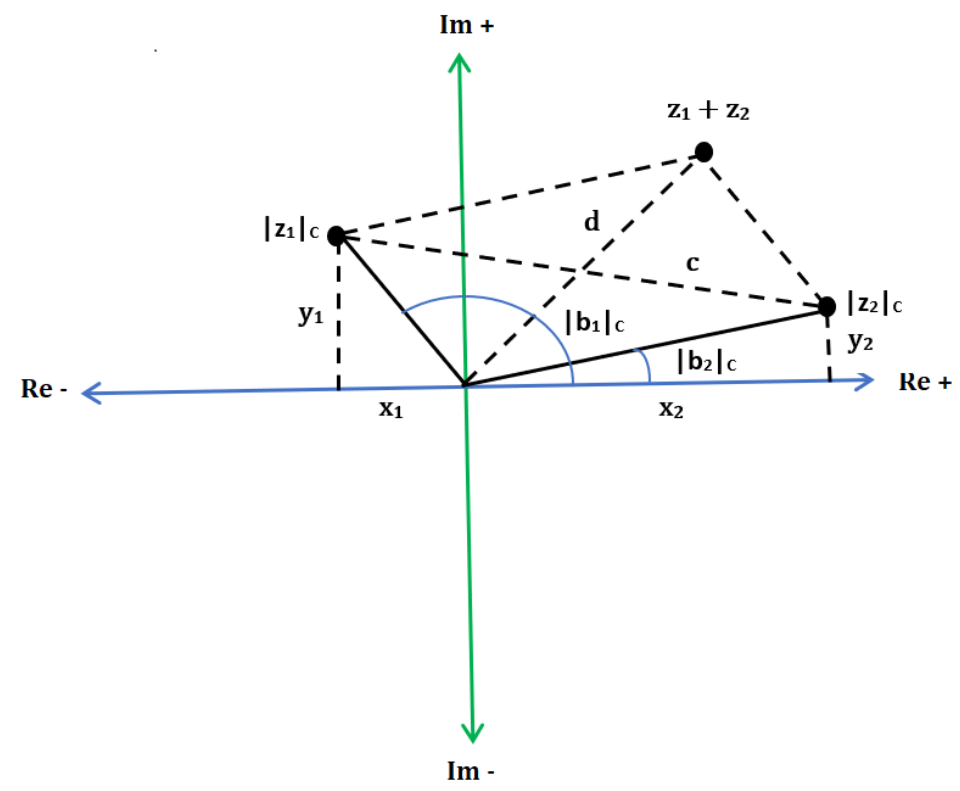

Figure 6: Addition and subtraction representation on the complex plane 


\subsection{Logarithmic representation}

The complex numbers in complete form are identified in a unique way by their real and imaginary arguments. Positioning the arguments coordinates on a Argand-Gauss diagram [6] is a possible representation. One can notice $(a ; b i)$ and $(a+b i)$ are equivalent notations for the coordinates, both are derived from the complete form $e^{a} e^{b i}$ or $e^{a+b i}$. Expressions at the exponent level only require $\mathbb{C}$ precision, thus all operations as defined in $\mathbb{C}$ can be used in an exponent. For example $-1 \cdot i=-i$ or $(-2)^{2}=4$, which both implicitly perfom a $\mathbb{C}$ truncation, can be used, the loss of precision will be without consequence. The number 0 is used in expressions as a normal number.

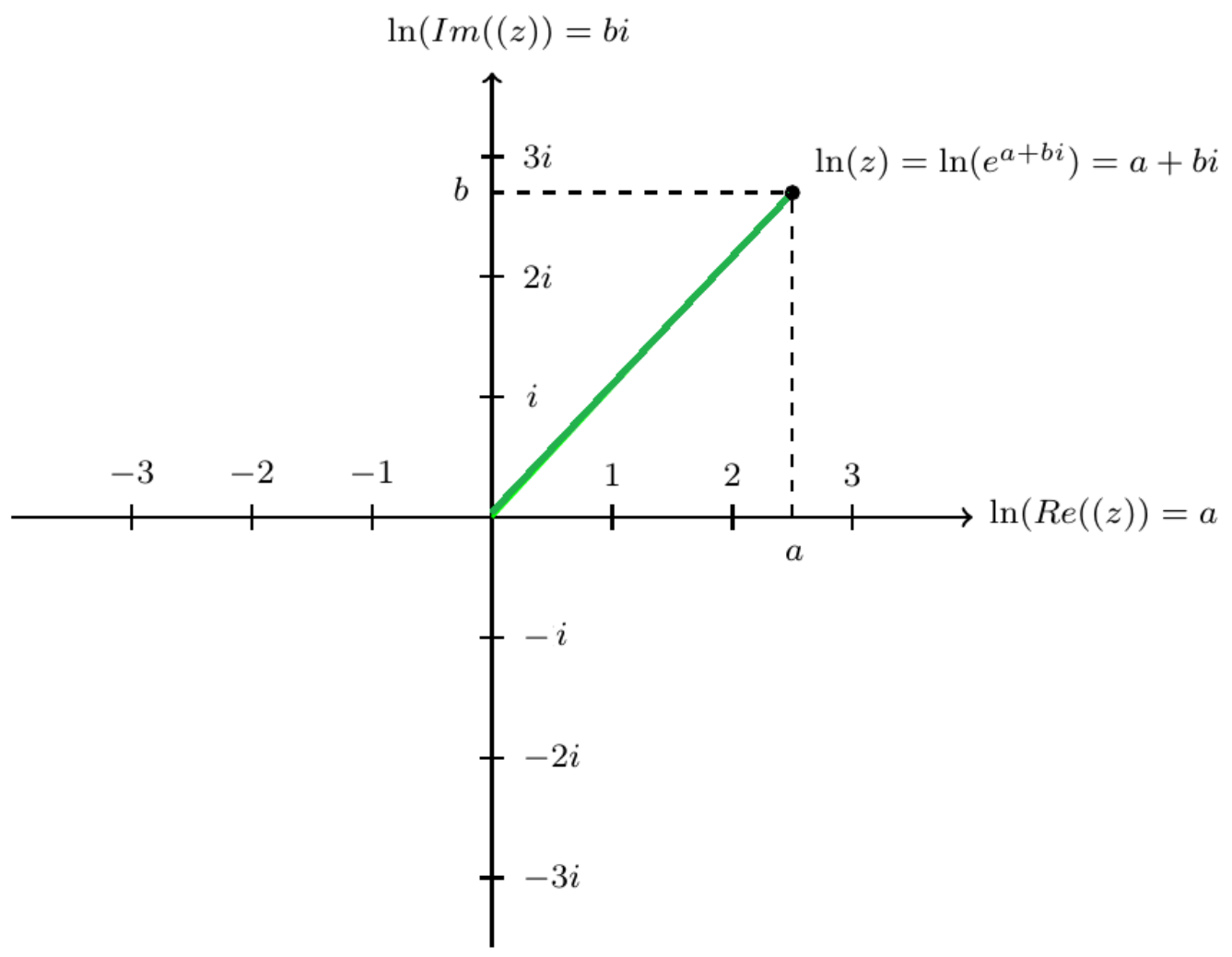

Figure 7: Logarithmic representation of $z=e^{a} e^{b i}$ on a Argand-Gauss diagram 


\section{$7 \quad$ Algebraic properties of the $\mathbb{E}$ number set}

From the properties of the real operations and functions composing a complex operation, it is possible to deduce the algebraic properties of $\mathbb{E}$. The properties of the value 0 are by convention inherited from $\mathbb{C}$ since the formulas 3.1 to 3.6 do not apply to that value.

\subsection{Commutativity}

The addition and the multiplication are the only commutative operations.

$z_{1} \times z_{2}=z_{2} \times z_{1}$

$z_{1}+z_{2}=z_{2}+z_{1}$

\subsection{Associativity}

The addition and the multiplication are the only associative operations.

$\left(z_{1}+z_{2}\right)+z_{3}=z_{1}+\left(z_{2}+z_{3}\right)$

$\left(z_{1} \times z_{2}\right) \times z_{3}=z_{1} \times\left(z_{2} \times z_{3}\right)$

\subsection{Distributivity}

The multiplication is distributive at $\mathbb{C}$ precision level over the addition and subtraction, the division is right distributive at $\mathbb{C}$ precision level over the addition and subtraction.

$$
\begin{gathered}
\left|z_{1} \times\left(z_{2}+z_{3}\right)\right|_{\mathbb{C}}=z_{1} \times z_{2}+z_{1} \times z_{3} \\
\left|z_{1} \times\left(z_{2}-z_{3}\right)\right|_{\mathbb{C}}=z_{1} \times z_{2}-z_{1} \times z_{3} \\
\left|\frac{z_{2}+z_{3}}{z_{1}}\right|_{\mathbb{C}}=\frac{z_{2}}{z_{1}}+\frac{z_{3}}{z_{1}} \\
\left|\frac{z_{2}-z_{3}}{z_{1}}\right|_{\mathbb{C}}=\frac{z_{2}}{z_{1}}-\frac{z_{3}}{z_{1}}
\end{gathered}
$$

Proof. Distributivity of multiplication over addition is $\mathbb{C}$ precise

Combining the multiplication and addition formulas 3.1 and 3.5, let $z_{1}=e^{a_{1}} e^{b_{1} i}, z_{2}=$ $e^{a_{2}} e^{b_{2} i}$ and $z_{3}=e^{a_{3}} e^{b_{3} i}$

$z=z_{1} \cdot\left(z_{2}+z_{3}\right)$

$a=a_{1}+\frac{1}{2} \ln \left(e^{2 a_{2}}+e^{2 a_{3}}+2 e^{a_{2}+a_{3}} \cos \left(b_{2}-b_{3}\right)\right)$

$b=b_{1}+\operatorname{Atan}\left(\frac{e^{a_{2}} \sin b_{2}+e^{a_{3}} \sin b_{3}}{e^{a_{2}} \cos b_{2}+e^{a_{3}} \cos b_{3}}\right)$

$z=z_{1} \cdot z_{2}+z_{1} \cdot z_{3}$

$a=\frac{1}{2} \ln \left(e^{2\left(a_{1}+a_{2}\right)}+e^{2\left(a_{1}+a_{3}\right)}+2 e^{\left(a_{1}+a_{2}\right)+\left(a_{1}+a_{3}\right)} \cos \left(\left(b_{1}+b_{2}\right)-\left(b_{1}+b_{3}\right)\right)\right)$

$=\frac{1}{2} \ln \left(e^{2 a_{1}}\left(e^{2 a_{2}}+e^{2 a_{3}}+2 e^{a_{2}+a_{3}} \cos \left(b_{2}-b_{3}\right)\right)\right)$

$=a_{1}+\frac{1}{2} \ln \left(e^{2 a_{2}}+e^{2 a_{3}}+2 e^{a_{2}+a_{3}} \cos \left(b_{2}-b_{3}\right)\right)$ 


$$
\begin{aligned}
& b=\operatorname{Atan}\left(\frac{e^{a_{1}+a_{2}} \sin \left(b_{1}+b_{2}\right)+e^{a_{1}+a_{3}} \sin \left(b_{1}+b_{3}\right)}{e^{a_{1}+a_{2}} \cos \left(b_{1}+b_{2}\right)+e^{a_{1}+a_{3}} \cos \left(b_{1}+b_{3}\right)}\right) \\
& =\operatorname{Atan}\left(\frac{e^{a_{2}}\left(\sin b_{1} \cos b_{2}+\cos b_{1} \sin b_{2}\right)+e^{a_{3}}\left(\sin b_{1} \cos b_{3}+\cos b_{1} \sin b_{3}\right)}{e^{a_{2}}\left(\cos b_{1} \cos b_{2}-\sin b_{1} \sin b_{2}\right)+e^{a_{3}}\left(\cos b_{1} \cos b_{3}-\sin b_{1} \sin b_{3}\right)}\right) \\
& =\operatorname{Atan}\left(\frac{\cos b_{1}\left(e^{a_{2}} \sin b_{2}+e^{a_{3}} \sin b_{3}\right)+\sin b_{1}\left(e^{a_{2}} \cos b_{2}+e^{a_{3}} \cos b_{3}\right)}{\cos b_{1}\left(e^{a_{2}} \cos b_{2}+e^{a_{3}} \cos b_{3}\right)-\sin b_{1}\left(e^{a_{2}} \sin b_{2}+e^{a_{3}} \sin b_{3}\right)}\right)
\end{aligned}
$$

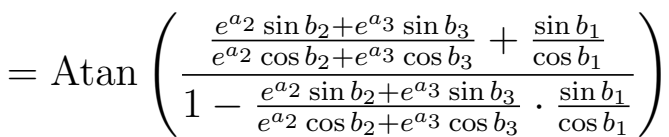

$$
\begin{aligned}
& =\operatorname{Atan}\left(\frac{\sin b_{1}}{\cos b_{1}}\right)+\operatorname{Atan}\left(\frac{e^{a_{2}} \sin b_{2}+e^{a_{3}} \sin b_{3}}{e^{a_{2}} \cos b_{2}+e^{a_{3}} \cos b_{3}}\right) \\
& =\left|b_{1}\right|_{\mathbb{C}}+\operatorname{Atan}\left(\frac{e^{a_{2}} \sin b_{2}+e^{a_{3}} \sin b_{3}}{e^{a_{2}} \cos b_{2}+e^{a_{3}} \cos b_{3}}\right)
\end{aligned}
$$

Example 14. Distributivity failure at $\mathbb{E}$ precision level

$$
\begin{aligned}
& -2 \cdot(i+1) \Longrightarrow e^{\ln 2} e^{\pi i} \cdot\left(e^{\frac{\pi}{2} i}+e^{0 i}\right)=e^{\ln 2} e^{\pi i} \cdot e^{\frac{1}{2} \ln 2} e^{\frac{\pi}{4} i}=e^{\frac{3}{2} \ln 2} e^{\frac{5 \pi}{4} i} \\
& (-2 \cdot i)+(-2 \cdot 1) \Longrightarrow e^{\ln 2} e^{\pi i} \cdot e^{\frac{\pi}{2} i}+e^{\ln 2} e^{\pi i} \cdot e^{0 i}=e^{\ln 2} e^{\frac{3 \pi}{2} i}+e^{\ln 2} e^{\pi i}=e^{\frac{3}{2} \ln 2} e^{-\frac{3 \pi}{4} i}
\end{aligned}
$$

In the first line the $\mathbb{E}$ precision is preserved because the final operation is a multiplication, in the second line the addition operates a $\mathbb{C}$ truncation, hence the results can be different.

\subsection{Identity element}

The identity element of addition and multiplication :

$$
\begin{aligned}
z_{1} \times e^{0} e^{0 i} & =z_{1} \\
z_{1}+0 & =z_{1}
\end{aligned}
$$

The right identity element of division and subtraction, exponentiation having an infinite set of right identities :

$$
\begin{aligned}
z_{1} / e^{0} e^{0 i} & =z_{1} \\
z_{1}-0 & =z_{1} \\
z_{1}{ }^{\left(e^{0} e^{2 k \pi i}\right)} & =z_{1} \quad(\text { with } k \in \mathbb{Z})
\end{aligned}
$$

\subsection{Inverse}

Multiplication, division and exponentiation are the exact reciprocal of their inverse operation :

$$
\begin{aligned}
& \frac{z_{1} \times z_{2}}{z_{1}}=z_{2} \\
& \frac{z_{2}}{z_{1}} \times z_{1}=z_{2}
\end{aligned}
$$


$z_{1}^{\log _{z 1}\left(z_{2}\right)}=z_{2}$

Logarithm, addition and subtraction are only the $\mathbb{C}$ precise reciprocal of their inverse operation:

$$
\begin{aligned}
\log _{z_{1}}\left(z_{1} z_{2}\right) & =\left|z_{2}\right|_{\mathbb{C}} \\
z_{2}+z_{1}-z_{1} & =\left|z_{2}\right|_{\mathbb{C}} \\
z_{2}-z_{1}+z_{1} & =\left|z_{2}\right|_{\mathbb{C}}
\end{aligned}
$$

Proof. Exponentiation is the exact inverse of logarithm

Using the logarithm formula 3.4 converted into algebraic form, let $z_{1}=e^{a_{1}} e^{b_{1} i}, z_{2}=e^{a_{2}} e^{b_{2} i}$ and $\log _{z_{1}}\left(z_{2}\right)=\frac{a_{1} a_{2}+b_{1} b_{2}}{a_{1}{ }^{2}+b_{1}{ }^{2}}+\frac{a_{1} b_{2}-a_{2} b_{1}}{a_{1}{ }^{2}+b_{1}{ }^{2}} i$

$$
\begin{aligned}
z & =z_{1}{ }^{\log _{z_{1}}\left(z_{2}\right)}=z_{2} \\
a & =\frac{a_{1}\left(a_{1} a_{2}+b_{1} b_{2}\right)-b_{1}\left(a_{1} b_{2}-b_{1} a_{2}\right)}{a_{1}^{2}+b_{1}{ }^{2}} \\
& =\frac{a_{1}{ }^{2} a_{2}+a_{1} b_{1} b_{2}-b_{1} a_{1} b_{2}+b_{1}{ }^{2} a_{2}}{a_{1}{ }^{2}+b_{1}{ }^{2}} \\
& =\frac{a_{2}\left(a_{1}^{2}+b_{1}{ }^{2}\right)}{a_{1}{ }^{2}+b_{1}{ }^{2}}=a_{2} \\
b & =\frac{b_{1}\left(a_{1} a_{2}+b_{1} b_{2}\right)+a_{1}\left(a_{1} b_{2}-b_{1} a_{2}\right)}{a_{1}{ }^{2}+b_{1}{ }^{2}} \\
& =\frac{b_{1} a_{1} a_{2}+b_{1}{ }^{2} b_{2}+a_{1}{ }^{2} b_{2}-a_{1} b_{1} a_{2}}{a_{1}{ }^{2}+b_{1}{ }^{2}} \\
& =\frac{b_{2}\left(a_{1}{ }^{2}+b_{1}{ }^{2}\right)}{a_{1}{ }^{2}+b_{1}{ }^{2}}=b_{2}
\end{aligned}
$$

\subsection{Symmetry}

$$
\begin{aligned}
e^{a} e^{b i} \cdot e^{-a} e^{-b i} & =e^{0} e^{0 i} \\
\frac{e^{a} e^{b i}}{e^{a} e^{b i}} & =e^{0} e^{0 i} \\
e^{a} e^{b i}+e^{a} e^{b i+(2 k+1) \pi i} & =0 \quad(\text { with } k \in \mathbb{Z}) \\
e^{a} e^{b i}-e^{a} e^{b i+2 k \pi i} & =0
\end{aligned}
$$

\subsection{Singularities}

At first we consider the singularities of operations where both operands are in $\mathbb{E} \backslash\{0\}$.

From the logarithm formula 3.4, one can easily deduce logarithms have a singularity when $z_{1}=e^{0} e^{0 i}$ and/or $z_{2}=e^{0} e^{0 i}$ caused by the division by 0 , the ln with operand 0 and the Atan with $0 / 0$ argument. Interestingly the singularities vanish if the operands are in the form $e^{0} e^{2 k \pi i}$ with $k \neq 0$.

$$
\log _{\left(e^{0} e^{0 i}\right)}\left(z_{2}\right)=\infty
$$




$$
\begin{aligned}
\log _{z_{1}}\left(e^{0} e^{0 i}\right) & =0 \\
\log _{\left(e^{0} e^{0 i}\right)}\left(e^{0} e^{0 i}\right) & =\text { undefined }
\end{aligned}
$$

From the formulas 3.5 and 3.6, it is possible to deduce both addition and subtraction have singularities caused by the ln with operand 0 and the Atan with $0 / 0$ argument.

$$
\begin{aligned}
e^{a} e^{b i}+e^{a} e^{b i+(2 k+1) \pi i} & =0 \quad(\text { with } k \in \mathbb{Z}) \\
e^{a} e^{b i}-e^{a} e^{b i+2 k \pi i} & =0
\end{aligned}
$$

The introduction of the element 0 allows to reduce some of the above singularities, but also adds new ones.

$$
\begin{aligned}
z_{1} \cdot 0 & =0 \\
z_{1} / 0 & =\infty \\
0 / z_{2} & =0 \\
0 / 0 & =\text { undefined } \\
z_{1}+0 & =z_{1} \\
z_{1}-0 & =z_{1} \\
0-z_{2} & =\left|z_{2} \cdot e^{\pi i}\right|_{\mathbb{C}} \\
0-0 & =0 \\
z_{1}^{0} & =e^{0} e^{0 i} \\
0^{z_{2}} & =0 \\
0^{0} & =e^{0} e^{0 i} \\
\log _{z_{1}}(0) & =\infty \\
\log _{0}\left(z_{2}\right) & =0 \\
\log _{0}(0) & =\text { undefined }
\end{aligned}
$$

In order to reduce the singularities, it is possible to include the infinite element such as $\mathbb{E}^{\prime}=\mathbb{E} \cup\{\infty\}$, and define the results of operations using $\infty$. However it would lead to new singularities such as $\infty-\infty$, whatever definition of $\mathbb{E}^{\prime}$ there will remain singularities that can ony be treated analytically.

\subsection{Algebraic structure of $\mathbb{E}$}

Conclusions can be made from formulas 3.1 to 3.6 and from the properties listed above :

- For each of the 6 complex operations, $\mathbb{E}$ has a closed algebraic structure, except for the singularities all results can be represented

- The multiplication and division maintain all their intrinsic properties such as in $\mathbb{C}$

- The addition and subtraction maintain all their intrinsic properties but only at $\mathbb{C}$ precision, since both operations do not require nor can provide any $\mathbb{E}$ precision

- The distributivity property generally only holds when the left side is truncated to $\mathbb{C}$ precision, thus distributivity is only $\mathbb{C}$ precise 
- The multiplication is clearly the defining operation and possess all the properties to constitute a multiplicative group $\left(\mathbb{E}^{*}, \cdot\right)$

- The field axioms are not all verified, since the addition/subtraction reciprocity and the distributivity do not hold exactly in $\mathbb{E}$

It would be a mistake to limit $\mathbb{E}$ to a multiplicative group, as many properties over the exponentiation and logarithm operations are added. All properties and identities hold to a certain extent, only limited by the operations maximum precision level. $\mathbb{E}$ is more to be considered as a complete number system.

\subsection{Properties comparison between $\mathbb{R}, \mathbb{C}$ and $\mathbb{E}$}

Table 3: Basic operations properties.

\begin{tabular}{lccc}
\hline Property & $\mathbb{R}$ & $\mathbb{C}$ & $\mathbb{E}$ \\
\hline Addition and subtraction closure & yes & yes & yes \\
Multiplication and division closure & yes & yes & yes \\
Addition and subtraction monovaluation & yes & yes & yes \\
Multiplication and division monovaluation & yes & yes & yes \\
Addition and multiplication commutativity & yes & yes & yes \\
Addition and multiplication associativity & yes & yes & yes \\
Multiplication distributivity over add/sub & yes & yes & $\mathbb{C}$ precise \\
Division right distributivity over add/sub & yes & yes & $\mathbb{C}$ precise \\
Identity element of add/sub & 0 & 0 & 0 \\
Identity element of mult/div & 1 & 1 & $e^{0} e^{0 i}=1$ \\
Addition/subtraction inverse & yes & yes & $\mathbb{C}$ precise \\
Multiplication/division inverse & yes & yes & yes \\
\hline
\end{tabular}

Table 4: Exponentiation and logarithm properties.

\begin{tabular}{lccc}
\hline Property & $\mathbb{R}$ & $\mathbb{C}$ & $\mathbb{E}$ \\
\hline Exponentiation $z_{1}^{z_{2}}$ closure & if $z_{1} \in \mathbb{R}_{+}$ & yes & yes \\
Logarithm $\log _{z_{1}}\left(z_{2}\right)$ closure & if $z_{1}, z_{2} \in \mathbb{R}_{+}$ & yes & yes \\
Exponentiation monovaluation & no & no & yes \\
Logarithm monovaluation & yes & no & yes \\
Exponentiation inverse of logarithm & & & \\
$z_{1} \log _{z 1}\left(z_{2}\right)=z_{2}$ & if $z_{1}, z_{2} \in \mathbb{R}_{+}$ & subset ${ }^{1}$ & yes \\
$\operatorname{Logarithm~inverse~of~exponentiation~}$ & & & \\
$\log _{z_{1}}\left(z_{1}{ }^{z_{2}}\right)=z_{2}$ & if $z_{1} \in \mathbb{R}_{+}$ & subset & $\mathbb{C}$ precise \\
\hline
\end{tabular}


1 The left side of the equation produces many more results, of which only a subset is equal to the right side. The equation always holds at principal value

Table 5: Exponentiation and logarithm identities.

Property
Exponent distributivity over multiplic.
$\left(z_{1} z_{2}\right)^{z_{3}}=z_{1} z_{3} z_{2} z_{3}$

Exponent distributivity over multiplic.

$\left(z_{1} z_{2}\right)^{z_{3}}=z_{1}^{z_{3}} z_{2}^{z_{3}}$

$$
\text { if } z_{1}, z_{2} \in \mathbb{R}_{+}
$$

no ${ }^{2}$

yes

Exponent distributivity over division

$$
\left(\frac{z_{1}}{z_{2}}\right)^{z_{3}}=\frac{z_{1} z_{3}}{z_{2} z_{3}}
$$

$$
\text { if } z_{1}, z_{2} \in \mathbb{R}_{+}
$$

no ${ }^{2}$

yes

Exponential product $z_{1}^{z_{2}} z_{1}^{z_{3}}=z_{1}^{z_{2}+z_{3}}$

$$
\text { if } z_{1} \in \mathbb{R}_{+}
$$

subset ${ }^{1}$

yes

Exponential quotient $\frac{z_{1} z_{2}}{z_{1} z_{3}}=z_{1}^{z_{2}-z_{3}}$

if $z_{1} \in \mathbb{R}_{+}$

subset ${ }^{1}$

yes

Exponential power $\left(z_{1}^{z_{2}}\right)^{z_{3}}=z_{1}^{z_{2} z_{3}}$

$$
\text { if } z_{1} \in \mathbb{R}_{+}
$$

no ${ }^{2}$

yes

Logarithm product

$\log _{z_{1}}\left(z_{2} z_{3}\right)=\log _{z_{1}}\left(z_{2}\right)+\log _{z_{1}}\left(z_{3}\right)$

$$
\text { if } z_{1}, z_{2}, z_{3} \in \mathbb{R}_{+}
$$

no ${ }^{2}$

yes

Logarithm quotient

$\log _{z_{1}}\left(\frac{z_{2}}{z_{3}}\right)=\log _{z_{1}}\left(z_{2}\right)-\log _{z_{1}}\left(z_{3}\right)$

if $z_{1}, z_{2}, z_{3} \in \mathbb{R}_{+}$

no ${ }^{2}$

yes

Logarithm power

$\log _{z_{1}}\left(z_{2} z_{3}\right)=z_{3} \log _{z_{1}}\left(z_{2}\right)$

if $z_{1}, z_{2} \in \mathbb{R}_{+}$

no ${ }^{2}$

$\mathbb{C}$ precise

Logarithm base substitution

$\log _{z_{1}}\left(z_{2}\right)=\frac{\log _{z_{3}}\left(z_{1}\right)}{\log _{z_{3}}\left(z_{2}\right)}$

if $z_{1}, z_{2}, z_{3} \in \mathbb{R}_{+}$

if $z_{3} \in \mathbb{R}_{+}$

$\mathbb{C}$ precise

${ }^{1}$ The left side of the equation produces many more results, of which only a subset is equal to the right side. The equation always holds at principal value

${ }^{2}$ Both sides of identities equations produce a different set of results, which are not necessarily equal at the principal value. Only when the imaginary argument remains within the $]-\pi ; \pi]$ boundary (from a $\mathbb{E}$ perspective) during each calculation step does the identity remain valid within a given branch

The exponential identities $z_{1}^{z_{2}} z_{1}^{z_{3}}=z_{1}^{z_{2}+z_{3}}$ and $\frac{z_{1} z_{2}}{z_{1} z_{3}}=z_{1}^{z_{2}-z_{3}}$ remain valid in $\mathbb{C}$ at the principal or at any other branch. The reason is clear, both identities never alter the exponentiation base, only the exponents are altered, as they do not require $\mathbb{E}$ precision no $\mathbb{C}$ truncation occurs. All other identities alter the exponentiation base or logarithm base or operand, thus any value outside the $]-\pi ; \pi]$ interval of the imaginary argument will trigger an identity failure. 


\section{Comments on the exponentiation and logarithm definition in $\mathbb{C}$}

The complex exponentiation in $\mathbb{C}$ is defined by the formula 1.1 , thus the primary multivalued result is in the form $e^{a+\alpha k \pi} e^{(b+\beta k \pi) i}$, where $a, \alpha, b, \beta \in \mathbb{R}$ and $k \in \mathbb{Z}$. To express the result in algebraic form a conversion is necessary, using the Euler formula the sine and cosine functions convert the result into algebraic form. However, during the conversion, precision is lost and the result principal value may be shifted. For example $(-1)^{3}=\left(e^{\ln (-1)}\right)^{3}=\left(e^{\pi i+2 k \pi i}\right)^{3}=e^{3 \pi i+6 k \pi i}$. Converting those values into algebraic form returns $-1=e^{\pi i+2 k \pi i}$, thus the principal value is reset to $e^{\pi i}$. Moreover there is no possibility to convert expressions such as $e^{6 k \pi i}$ without loss of information. In the example $\left(i^{-5}\right)^{i}$, when the result of $i^{-5}$ is reconverted into algebraic form the principal value result is shifted from $e^{-\frac{5 \pi i}{2}}$ to $e^{-\frac{\pi i}{2}}$, the result of $\left(i^{-5}\right)^{i}$ becoming $e^{\frac{\pi}{2}}$ instead of $e^{\frac{5 \pi}{2}}$.

The multivaluation of the complex exponentiation is not induced by the logarithm, but by the algebraic form of the base. Since no identity is available to exploit the base as such, the formula 1.1 is equivalent as substituting the base by an infinity of bases, the so-called exponential form, using the formula $z=|z| e^{\arg (z) i}=|z| e^{\theta i+2 k \pi i}$. In general the multivaluation is assumed, unless explicitly restricting the exponentiation to real positives with notations such as $|z|^{\alpha}$ or $\sqrt{a^{2}+b^{2}}$ which both assume a single valued real positive base. Expressions such as $\left(e^{\pi i}\right)^{\frac{1}{3}}$ return a single valued result, unless $e^{\pi i}$ is converted into algebraic form, in which case $\left(e^{\pi i}\right)^{\frac{1}{3}} \Longrightarrow(-1)^{\frac{1}{3}}=\left(e^{\pi i+2 k \pi i}\right)^{\frac{1}{3}}$

The complex logarithm as defined by L. Euler [1] is restricted to the base $e$ or at least to real positive values. Euler himself does not mention a multivalued logarithm function, rather he speaks of each real or complex number having an infinite number of logarithms. Indeed, as for the exponentiation base, the logarithm operand cannot be exploited directly in algebraic form, thus has to be converted into exponential form, $\ln (z)=\ln \left(|z| e^{\arg (z) i}\right)=\ln \left(|z| e^{\theta i+2 k \pi i}\right)=\ln |z|+\theta i+2 k \pi i$. The primary result being in algebraic form, no conversion is required nor any loss of precision is induced. The multivaluation is solely induced by the operand substitution, for example $\ln (1)=2 k \pi i$ and $\ln (-1)=\pi i+2 k \pi i$. On the other hand $\ln |z|$ is assumed single valued as the operand is implicitly substituted by $x e^{0 i}$. Expressions such as $\ln \left(e^{\pi i}\right)=\pi i$ are single valued, unless $e^{\pi i}$ is converted to algebraic form, in which case $\ln \left(e^{\pi i}\right) \Longrightarrow \ln (-1)=\pi i+2 k \pi i$.

Notations such as $\ln , \log _{2}$ or $\log _{10}$ assume the logarithm base is in the form $x e^{0 i}$. For bases the same logic applies as for the exponentiation, a base in algebraic form can be substituted by the equivalent exponential form, or by any particular value in complete form. As an example, for $\log _{-1}$ the base can be assumed as monovalued $e^{\pi i}$ or multivalued $e^{\pi i+2 k \pi i}$.

It is clear there is only one unique exponentiation and one unique logarithm complex operation. The different notations conventions and different assumptions regarding the operands substitutions are creating some confusion, which can be blamed on the lack of precision of the algebraic form. In complete form, real positive numbers are not fundamentally different, all operands are in the form $e^{a} e^{b i}$, moreover the concepts of principal value and branches are no longer necessary. 
When dealing with exponentiation and logarithm in $\mathbb{C}$ the equality $e^{\alpha i}=e^{\alpha i+2 k \pi i}$ is automatically assumed, by an analogy with the trigonometric circle where an angle of $\alpha$ is equal to $\alpha+2 k \pi$. However from a $\mathbb{E}$ perspective only $\left|e^{\alpha i}\right|_{\mathbb{C}}=\left|e^{\alpha i+2 k \pi i}\right|_{\mathbb{C}}$ is valid. The formulas $\cos (\alpha)+i \sin (\alpha)=\cos (\alpha+2 k \pi)+i \sin (\alpha+2 k \pi)=\sum_{n=0}^{\infty} \frac{(\alpha i)^{n}}{n !}=\sum_{n=0}^{\infty} \frac{(\alpha i+2 k \pi i)^{n}}{n !}$ are strictly equal, but are also equal in deconstructing the complete form and reconstructing a result in algebraic form, as such they literally truncate the precision of the complete form.

\section{Conclusion}

As demonstrated in this article, the complex exponentiation base and result, the complex logarithm base and operand cannot be represented precisely in algebraic form. The same observation holds for the multiplication and division results when used in combination with an exponentiation or logarithm. For this reason alone, multivalued results, identity failures and even wrong results are obtained when computing exclusively in $\mathbb{C}$.

The establishment of the complete form is an attempt to restore the properties of exponentiation and logarithm, and to ease the conceptualization and handling of both operations when all operands are complex. Moreover the $\mathbb{E}$ set of complex numbers in complete form can be viewed as a "natural" extension of $\mathbb{C}$. Within the sequence $\mathbb{N} \subset \mathbb{Z} \subset \mathbb{R} \subset \mathbb{C} \subset \mathbb{E}$ each set extends the capacity of the predecessor set by providing new elements, thus new symbolic representations of numbers. Each element in a given set is uniquely linked to a predecessor set element through an equivalence relation, therefore an element can always be truncated to the predecessor set precision level. Similarly the geometric representations are extended while preserving the predecessor sets representations.

Labelling expressions such as $e^{a} e^{b i}$ as numbers might seem strange, though we believe it is totally justified by the extra precision and possibilities they introduce, as they overcome some limitations encountered in $\mathbb{C}$ with the algebraic form. As we have frequently illustrated with examples, it remains possible to combine the algebraic and complete form inside expressions and formulas. Though outside the $]-\pi ; \pi]$ boundary of the imaginary argument, the elementary algebra rules and formulas cannot be applied as such, and should be replaced by the formulas 3.1 to 3.6 .

\section{Author :}

Daniel Tischhauser

Independent researcher, Geneva, Switzerland

Email : dtischhauser.math@gmail.com

\section{References}

[1] Euler, Leonhard; De la controverse entre Mrs. Leibnitz et Bernoulli sur les logarithmes des nombres négatifs et imaginaires. 1749, 165-178 . 
[2] Euler, Leonhard; Recherches sur les racines imaginaires des équations. 1749, 272-276.

[3] Ohm, Martin; Systems der Mathematik, Theil 2, 1829, 426-443.

[4] Cajori, Florian; History of the Exponential and Logarithmic Concepts. The American Mathematical Monthly 1913, vol. 20, no. 6, 173-182.

[5] Steiner J., Clausen T., Abel Niels Henrik; Aufgaben und Lehrsätze, erstere aufzulösen, letztere zu beweisen. Journal für die reine und angewandte Mathematik 1827, vol. 2, $286-287$.

[6] Argand, Jean-Robert; Essai sur une manière de représenter les quantités imaginaires dans les constructions géométriques, Gauthier-Villars, 1874.

[7] Shilov Georgi E.; Elementary Real and Complex Analysis, revised english edition; Dover publications Inc., 1996.

[8] Nahin, Paul J.; An Imaginary Tale, the story of $\sqrt{-1}$, Princeton University Press, 2016. 